\title{
Synthesis and Polymerization of Mono-p-alkoxy-Substituted Triphenylmethyl Methacrylate
}

\author{
Yusuke KaWAKami, ${ }^{*}$ Yutaka SaKaI, and Akihiko OKada \\ Department of Synthetic Chemistry, School of Engineering, \\ Nagoya University, Chikusa, Nagoya 464, Japan
}

(Received February 15, 1990)

\begin{abstract}
Triphenylmethyl methacrylates having alkoxy groups, or mesogenic groups with alkyleneoxy spacer, as $p$-substituents of one of the phenyl groups were synthesized. Although the monomers and polymers were considerably unstable against hydrolysis, polymers could be obtained by radical polymerization by careful handling of the compounds. Anionic initiators, such as butylithium, usually failed to give high polymers. This seems to be because of the difficulty in obtaining completely dry monomers for anionic polymerization. Triad isotacticity of the polymers was evaluated after converting the polymers into poly(methyl methacrylate)s. Polymers obtained not only by anionic polymerization but also by radical polymerization showed considerably high isotacticity. The effects of the substituents on the isotacticity of the polymers are described.
\end{abstract}

KEY WORDS Triphenylmethyl Metacrylate / Radical Polymerization /

Anionic Polymerization / Tacticity / Poly(methyl methacrylate) / Mesogen /

Triphenylmethyl methacrylate is reported to give polymers of considerably high triad isotacticity even by radical initiators. This was attributed to the stabilization of $3_{1}$ helical structure by steric and polar interaction between phenyl groups of the triphenylmethyl group along the main chain. ${ }^{1}$ The monomer is also reported to give an optically active polymer by an initiator system composed of alkyllithium and optically active amine like sparteine. The formed optically active polymer can maintain helical conformation in one handed sense, even in solvent, stabilized by bulky triphenylmethyl groups along the highly isotactic polymer main chain. ${ }^{2-4}$ Stabilization of such helical structure of a vinyl polymer by long side chains is of great interest in connection with the origin of cholesteric liquid crystalline phase of $\operatorname{poly}(\gamma$ alkyl-L-glutamate) which maintain right handed helical structure in concentrated solution (alkyl = benzyl: lyotropic liquid crystalline) or in solid state (alkyl=long alkyl chain: thermotropic liquid crystalline). ${ }^{5,6}$
In order to study the possibility of the stabilization of the helical structure of a vinyl polymer by long alkoxy substituents as tail groups, we chose mono-p-alkoxy-substituted triphenylmethyl methacrylate as a monomer, and studied the synthetic method of introduction of long alkoxy groups to the para position of one of the phenyl group of the monomer, the polymerizability of these monomers, and the structure of formed polymers specially paying attention to the effects of the tail groups on the isotacticity of the polymer main chain.

\section{EXPERIMENTAL}

\section{General}

${ }^{1} \mathrm{H}$ NMR spectra were obtained on a Varian NMR spectrometer model Gemini 200.

\section{Synthesis of Monomers}

The reaction scheme of the synthesis of the monomers is shown in Scheme 1. 


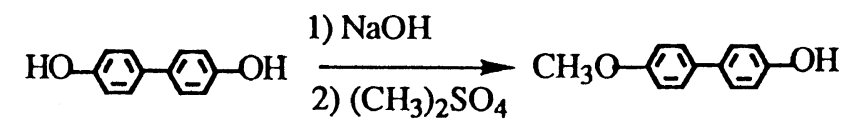

1) NaH, DMF

2) $\overrightarrow{\mathrm{Br}\left(\mathrm{CH}_{2}\right)_{\mathrm{n}} \mathrm{Br}}$

$\mathrm{CH}_{3} \mathrm{O}-\mathrm{O}\left(\mathrm{CH}_{2}\right)_{\mathrm{B}} \mathrm{Br}$
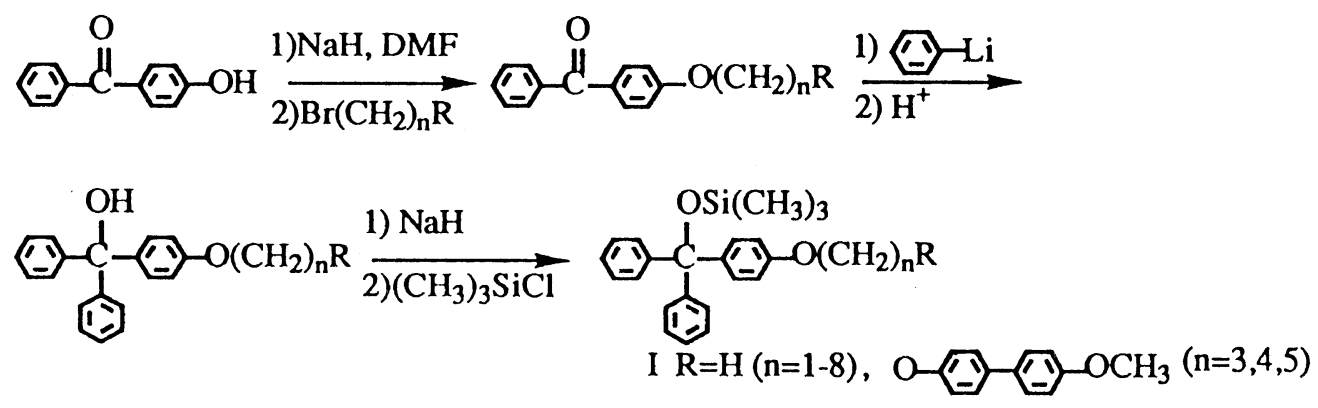<smiles>C=C(C)C(=O)OC</smiles>

II<smiles>[R2]CCOC(=O)C(C(=O)OCC)(c1ccccc1)c1ccc(OCC[R])cc1</smiles>

$\mathrm{R}=\mathrm{H}(\mathrm{n}=1-8), \mathrm{O}-\mathrm{O}-\mathrm{OCH}_{3} \quad(\mathrm{n}=3,4,5)$

Scheme 1. Synthetic routes to monomers.

4-( $\omega$-Bromoalkoxy)-4'-methoxybiphenyls

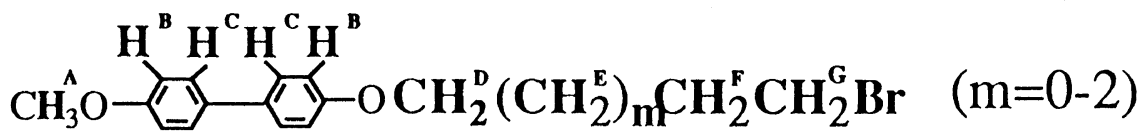

4-(4-Bromobutoxy)-4'-methoxybiphenyl, 4-(3-bromopropoxy)-4'-methoxybiphenyl and 4-(5-bromopentyloxy)-4'-methoxybiphenyl were synthesized similarly. A typical example is given.

4-(4-Bromobutoxy)-4'-methoxybiphenyl $(m=$ 1) To sodium hydride $(0.36 \mathrm{~g}, 15.0 \mathrm{mmol})$ in dimethylformamide $(50 \mathrm{ml})$, 4-methoxy-4'-hydroxybiphenyl $(2.00 \mathrm{~g}, 9.99 \mathrm{mmol})$ was added and the reaction system is stirred for $10 \mathrm{~min}$. To this reaction mixture, large excess of 1,4-dibromobutane $(25 \mathrm{~g}, 115.8 \mathrm{mmol})$ was added and the whole system was stirred for further $12 \mathrm{~h}$. After the solvent and excess 1,4-dibromobutane were removed by evaporation under vacuum, chloroform $(300 \mathrm{ml})$ and aqueous sodium hydroxide $(0.1 \mathrm{~N}, 100 \mathrm{ml})$ were mixed with the system and organic layer was separated. This process assured the complete removal of the excess hydroxybiphenyl. Evaporation of the solvent after drying over magnesium sulfate gave crude product. The crude product was recrystallized from ethanol.

Yield $86 \%$. mp $124.9^{\circ} \mathrm{C}$.

Chemical shifts $\delta \quad 1.80-2.32 \quad(\mathrm{~m}, 4 \mathrm{H}$, $\left.J=6.0 \mathrm{~Hz}, \mathrm{H}^{\mathrm{E}}, \mathrm{H}^{\mathrm{F}}\right), 3.48\left(\mathrm{t}, 2 \mathrm{H}, J=6.0 \mathrm{~Hz}, \mathrm{H}^{\mathrm{G}}\right)$, $3.83\left(\mathrm{~s}, 3 \mathrm{H}, \mathrm{H}^{\mathrm{A}}\right), 4.00\left(\mathrm{t}, 2 \mathrm{H}, J=6.0 \mathrm{~Hz}, \mathrm{H}^{\mathrm{D}}\right)$, $6.93\left(\mathrm{~d}, 4 \mathrm{H}, J=8.0 \mathrm{~Hz}, \mathrm{H}^{\mathrm{B}}\right), 7.45(\mathrm{~d}, 4 \mathrm{H}$, 
$\left.J=8.0 \mathrm{~Hz}, \mathrm{H}^{\mathrm{C}}\right)$.

4-(3-Bromopropoxy)-4'-methoxybiphenyl $(m=0)$

Yield $83.0 \%$. mp $97.7^{\circ} \mathrm{C}$.

Chemical shifts $\delta 2.35$ (quintet, $2 \mathrm{H}, J=6.0$ $\left.\mathrm{Hz}, \mathrm{H}^{\mathrm{F}}\right), 3.64\left(\mathrm{t}, 2 \mathrm{H}, J=6.0 \mathrm{~Hz}, \mathrm{H}^{\mathrm{G}}\right), 3.84(\mathrm{~s}$, $\left.3 \mathrm{H}, \mathrm{H}^{\mathrm{A}}\right), 4.15\left(\mathrm{t}, 2 \mathrm{H}, J=6.0 \mathrm{~Hz}, \mathrm{H}^{\mathrm{D}}\right), 6.95(\mathrm{~d}$, $\left.4 \mathrm{H}, J=8.0 \mathrm{~Hz}, \mathrm{H}^{\mathrm{B}}\right), 7.48(\mathrm{~d}, 4 \mathrm{H}, J=8.0 \mathrm{~Hz}$, $\mathrm{H}^{\mathrm{C}}$.

4-Alkoxybenzophenones

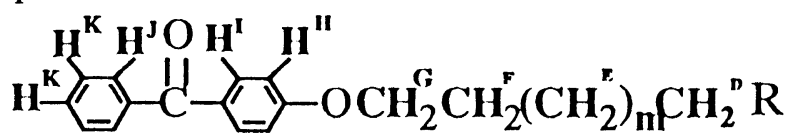

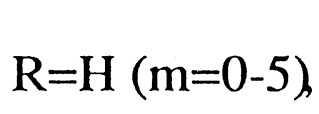

A typical example is given.

4-Butoxybenzophenone $(\mathrm{R}=\mathrm{H}, m=1)$. To sodium hydride $(0.67 \mathrm{~g}, 27.7 \mathrm{mmol})$ in dimethylformamide $(50 \mathrm{ml})$ 4-hydroxybenzophenone $(5.00 \mathrm{~g}, 25.2 \mathrm{mmol})$ was added portionwise, and the reaction mixture was stirred for $1 \mathrm{~h}$. Butyl bromide $(4.19 \mathrm{~g}, 30.6 \mathrm{mmol})$ was added and the system was stirred for $20 \mathrm{~h}$ at $70^{\circ} \mathrm{C}$. After the system was cooled down to room temperature, excess butyl bromide and dimethylformamide were removed by evaporation. Residual solid was dissolved in ether $(200 \mathrm{ml})$ and washed with dilute aqueous sodium hydroxide $(0.1 \mathrm{~N}, 100 \mathrm{ml})$. The organic layer was dried over magnesium sulfate, and the evaporation of the solvent gave product. The crude product was purified on silica gel column chromatography (eluent: hexaneether, $4: 1, R f=0.28$ ).

Yield $90.5 \%$. mp $32.4^{\circ} \mathrm{C}$.

Chemical shifts $\delta 0.97\left(\mathrm{t}, 3 \mathrm{H}, J=7.0 \mathrm{~Hz}, \mathrm{H}^{\mathrm{D}}\right)$, $1.16-2.08\left(\mathrm{~m}, 4 \mathrm{H}, \mathrm{H}^{\mathrm{E}}, \mathrm{H}^{\mathrm{F}}\right), 4.03(\mathrm{t}, 2 \mathrm{H}$, $\left.J=7.0 \mathrm{~Hz}, \mathrm{H}^{\mathrm{G}}\right), 6.93\left(\mathrm{~d}, 2 \mathrm{H}, J=9.0 \mathrm{~Hz}, \mathrm{H}^{\mathrm{H}}\right)$, 7.09-7.96 (m, 5H, $\left.\mathrm{H}^{\mathrm{J}}, \mathrm{H}^{\mathrm{K}}\right), 7.81(\mathrm{~d}, 2 \mathrm{H}$, $\left.J=9.0 \mathrm{~Hz}, \mathrm{H}^{\mathrm{I}}\right)$.

Other 4-alkoxybenzophenones were synthesized similarly to 4-butoxybenzophenone. They are also solid, and purified similarly. 4Methoxybenzophenone and 4-ethoxybenzo-
4-(5-Bromopentyloxy)-4'-methoxybiphenyl

$(m=2)$

Yield $90 \% . \mathrm{mp} 84.1^{\circ} \mathrm{C}$.

Chemical shifts $\delta 1.25-2.30\left(\mathrm{~m}, 6 \mathrm{H}, \mathrm{H}^{\mathrm{E}}\right.$, $\left.\mathrm{H}^{\mathrm{F}}\right), 3.43\left(\mathrm{t}, 2 \mathrm{H}, J=6.0 \mathrm{~Hz}, \mathrm{H}^{\mathrm{G}}\right), 3.82(\mathrm{~s}, 3 \mathrm{H}$, $\left.\mathrm{H}^{\mathrm{A}}\right), 3.99\left(\mathrm{t}, 2 \mathrm{H}, J=6.0 \mathrm{~Hz}, \mathrm{H}^{\mathrm{D}}\right), 6.92(\mathrm{~d}, 4 \mathrm{H}$, $\left.J=8.0 \mathrm{~Hz}, \mathrm{H}^{\mathrm{B}}\right), 7.46\left(\mathrm{~d}, 4 \mathrm{H}, J=8.0 \mathrm{~Hz}, \mathrm{H}^{\mathrm{C}}\right)$. phenone were also synthesized.

4-Methoxybenzophenone. Methyl iodide was used as an alkylating agent.

Yield quant. $\mathrm{mp} 57.4^{\circ} \mathrm{C}$.

Chemical shifts $\delta 3.90\left(\mathrm{~s}, 3 \mathrm{H}, \mathrm{CH}_{3}\right), 6.97$ (d, $\left.2 \mathrm{H}, J=9.0 \mathrm{~Hz}, \mathrm{H}^{\mathrm{H}}\right), 7.42-7.63\left(\mathrm{~m}, 3 \mathrm{H}, \mathrm{H}^{\mathrm{K}}\right)$, 7.77-7.87 (m, $\left.4 \mathrm{H}, \mathrm{H}^{\mathrm{I}}, \mathrm{H}^{\mathrm{J}}\right)$.

\section{4-Ethoxybenzophenone.}

Yield $75.4 \% . \mathrm{mp} 46.3^{\circ} \mathrm{C} . R f=0.21$ (hexane-ether, $5: 1)$.

Chemical shifts $\delta 1.43(\mathrm{t}, 3 \mathrm{H}, J=7.0 \mathrm{~Hz}$, $\left.\mathrm{CH}_{3}\right), 4.10$ (q, 2H,J=7.0 Hz, $\left.\mathrm{CH}_{2}\right), 6.92$ (d, $\left.2 \mathrm{H}, J=9.0 \mathrm{~Hz}, \mathrm{H}^{\mathrm{H}}\right), 7.38-7.60\left(\mathrm{~m}, 3 \mathrm{H}, \mathrm{H}^{\mathrm{K}}\right)$, $7.68-7.80\left(\mathrm{~m}, 2 \mathrm{H}, \mathrm{H}^{\mathrm{J}}\right), 7.80(\mathrm{~d}, 2 \mathrm{H}, J=9.0 \mathrm{~Hz}$, $\left.\mathrm{H}^{\mathrm{l}}\right)$.

4-Propoxybenzophenone $(\mathrm{R}=\mathrm{H}, m=0)$.

Yield $95.8 \% . \mathrm{mp} 64.3^{\circ} \mathrm{C} . R f=0.28$ (hexane-ether, $5: 1$ ).

Chemical shifts $\delta 1.03\left(\mathrm{t}, 3 \mathrm{H}, J=7.0 \mathrm{~Hz}, \mathrm{H}^{\mathrm{D}}\right)$, $1.82\left(\mathrm{~m}, 2 \mathrm{H}, \mathrm{H}^{\mathrm{F}}\right), 3.98\left(\mathrm{t}, 2 \mathrm{H}, J=7.0 \mathrm{~Hz}, \mathrm{H}^{\mathrm{G}}\right)$, $6.93\left(\mathrm{~d}, 2 \mathrm{H}, J=9.0 \mathrm{~Hz}, \mathrm{H}^{\mathrm{H}}\right), 7.39-7.61(\mathrm{~m}$, $\left.3 \mathrm{H}, \mathrm{H}^{\mathrm{K}}\right), 7.68-7.77\left(\mathrm{~m}, 2 \mathrm{H}, \mathrm{H}^{\mathrm{J}}\right), 7.80(\mathrm{~d}, 2 \mathrm{H}$, $\left.J=9.0 \mathrm{~Hz}, \mathrm{H}^{\mathrm{I}}\right)$.

4-Pentyloxybenzophenone $(\mathrm{R}=\mathrm{H}, m=2)$.

Yield $76.9 \% . \mathrm{mp} 32.8^{\circ} \mathrm{C} . R f=0.31$ (hexane-ether, $5: 1$ ).

Chemical shifts $\delta 0.92\left(\mathrm{t}, 3 \mathrm{H}, J=7.0 \mathrm{~Hz}, \mathrm{H}^{\mathrm{D}}\right)$, $1.27-1.55\left(\mathrm{~m}, 4 \mathrm{H}, \mathrm{H}^{\mathrm{E}}\right), 1.80$ (quint, $J=7.0 \mathrm{~Hz}$, $\left.2 \mathrm{H}, \mathrm{H}^{\mathrm{F}}\right), 4.02\left(\mathrm{t}, 2 \mathrm{H}, J=7.0 \mathrm{~Hz}, \mathrm{H}^{\mathrm{G}}\right), 6.93(\mathrm{~d}$, 
$\left.2 \mathrm{H}, J=9.0 \mathrm{~Hz}, \mathrm{H}^{\mathrm{H}}\right), 7.38-7.61\left(\mathrm{~m}, 3 \mathrm{H}, \mathrm{H}^{\mathrm{K}}\right)$, $7.68-7.78\left(\mathrm{~m}, 2 \mathrm{H}, \mathrm{H}^{\mathrm{J}}\right), 7.80(\mathrm{~d}, 2 \mathrm{H}, J=9.0 \mathrm{~Hz}$, $\left.\mathrm{H}^{\mathrm{I}}\right)$.

4-Hexyloxybenzophenone $(\mathrm{R}=\mathrm{H}, m=3)$.

Yield $70.3 \% . \mathrm{mp} 33.8^{\circ} \mathrm{C} . R f=0.26$ (hexane-ether, $5: 1$ ).

Chemical shifts $\delta 0.89\left(\mathrm{t}, 3 \mathrm{H}, J=7.0 \mathrm{~Hz}, \mathrm{H}^{\mathrm{D}}\right)$, $1.20-1.58\left(\mathrm{~m}, 6 \mathrm{H}, \mathrm{H}^{\mathrm{E}}\right), 1.79$ (quint, $2 \mathrm{H}$, $\left.J=7.0 \mathrm{~Hz}, \mathrm{H}^{\mathrm{F}}\right), 4.02\left(\mathrm{t}, 2 \mathrm{H}, J=7.0 \mathrm{~Hz}, \mathrm{H}^{\mathrm{G}}\right)$, $6.93\left(\mathrm{~d}, 2 \mathrm{H}, J=9.0 \mathrm{~Hz}, \mathrm{H}^{\mathrm{H}}\right), 7.38-7.61(\mathrm{~m}$, $\left.3 \mathrm{H}, \mathrm{H}^{\mathrm{K}}\right), 7.68-7.78\left(\mathrm{~m}, 2 \mathrm{H}, \mathrm{H}^{\mathrm{J}}\right), 7.80(\mathrm{~d}, 2 \mathrm{H}$, $\left.J=9.0 \mathrm{~Hz}, \mathrm{H}^{\mathrm{I}}\right)$.

4-Heptyloxybenzophenone $(\mathrm{R}=\mathrm{H}, m=4)$.

Yield $48.2 \% . \mathrm{mp} 46.6^{\circ} \mathrm{C} . R f=0.36$ (hexane-ether, $5: 1$ ).

Chemical shifts $\delta 0.87\left(\mathrm{t}, 3 \mathrm{H}, J=7.0 \mathrm{~Hz}, \mathrm{H}^{\mathrm{D}}\right)$, $1.28-1.57\left(\mathrm{~m}, 8 \mathrm{H}, \mathrm{H}^{\mathrm{E}}\right), 1.79$ (quint, $2 \mathrm{H}$, $\left.J=7.0 \mathrm{~Hz}, \mathrm{H}^{\mathrm{F}}\right), 4.01\left(\mathrm{t}, 2 \mathrm{H}, J=7.0 \mathrm{~Hz}, \mathrm{H}^{\mathrm{G}}\right)$, $6.92\left(\mathrm{~d}, 2 \mathrm{H}, J=9.0 \mathrm{~Hz}, \mathrm{H}^{\mathrm{H}}\right), 7.39-7.61(\mathrm{~m}$, $\left.3 \mathrm{H}, \mathrm{H}^{\mathrm{K}}\right), 7.69-7.79\left(\mathrm{~m}, 2 \mathrm{H}, \mathrm{H}^{\mathrm{J}}\right), 7.80(\mathrm{~d}, 2 \mathrm{H}$, $\left.J=9.0 \mathrm{~Hz}, \mathrm{H}^{\mathrm{I}}\right)$.

4-Octyloxybenzophenone $(\mathrm{R}=\mathrm{H}, m=5)$.

Yield $65.4 \% . \mathrm{mp} 43.8^{\circ} \mathrm{C} . R f=0.43$ (hexane-ether, $4: 1$ ).

Chemical shifts $\delta 0.88\left(\mathrm{t}, 3 \mathrm{H}, J=7.0 \mathrm{~Hz}, \mathrm{H}^{\mathrm{D}}\right)$, $1.05-2.13\left(\mathrm{~m}, 12 \mathrm{H}, \mathrm{H}^{\mathrm{E}}, \mathrm{H}^{\mathrm{F}}\right), 4.02(\mathrm{t}, 2 \mathrm{H}$, $\left.J=7.0 \mathrm{~Hz}, \mathrm{H}^{\mathrm{G}}\right), 6.93\left(\mathrm{~d}, 2 \mathrm{H}, J=9.0 \mathrm{~Hz}, \mathrm{H}^{\mathrm{H}}\right)$, 7.09-7.96 (m, 5H, $\left.\mathrm{H}^{\mathrm{J}}, \mathrm{H}^{\mathrm{K}}\right), 7.81(\mathrm{~d}, 2 \mathrm{H}$, $\left.J=9.0 \mathrm{~Hz}, \mathrm{H}^{\mathrm{l}}\right)$.

4-\{3-(4-Methoxy-4'-oxybiphenyl)propoxy\}benzophenone, 4-\{4-(4-methoxy-4'-oxybiphenyl)butoxy $\}$ benzophenone, and 4-\{5-(4methoxy-4'-oxybiphenyl)pentyloxy\}benzo-

(4-Alkoxyphenyl)diphenylmethanols

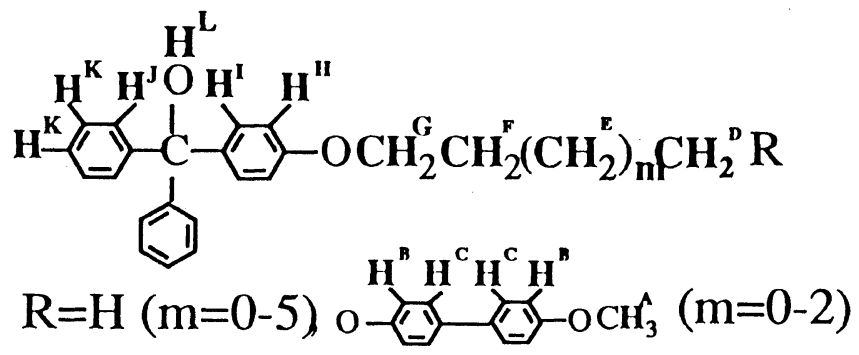

(4-Alkoxyphenyl)diphenylmethanols were synthesized from 4-alkoxybenzophenones by the reaction with phenyllithium in ether or phenone were synthesized similarly to 4-butoxybenzophenone.

4-\{3-(4-Methoxy-4'-oxybiphenyl)propoxy $\}$ benzophenone ( $\mathrm{R}=4$-methoxy-4'-oxybiphenyl, $m=0$ ).

Recrystallized from THF. mp $146.3^{\circ} \mathrm{C}$. Yield $65.4 \%$.

Chemical shifts $\delta 2.33$ (quint, $2 \mathrm{H}, J=7.0 \mathrm{~Hz}$, $\left.\mathrm{H}^{\mathrm{F}}\right), 3.84\left(\mathrm{~s}, 3 \mathrm{H}, \mathrm{H}^{\mathrm{A}}\right), 4.15-4.32(\mathrm{dt}, 4 \mathrm{H}$, $\left.J=7.0 \mathrm{~Hz}, \mathrm{H}^{\mathrm{D}}, \mathrm{H}^{\mathrm{G}}\right), 6.90-7.04$ (three d, $6 \mathrm{H}$, $\left.J=9.0 \mathrm{~Hz}, \mathrm{H}^{\mathrm{B}}, \mathrm{H}^{\mathrm{H}}\right), 7.42-7.63\left(\mathrm{~m}, 3 \mathrm{H}, \mathrm{H}^{\mathrm{K}}\right)$, $7.48\left(\mathrm{~d}, 4 \mathrm{H}, J=9.0 \mathrm{~Hz}, \mathrm{H}^{\mathrm{C}}\right), 7.72-7.78(\mathrm{~m}, 4 \mathrm{H}$, $\left.J=9.0 \mathrm{~Hz}, \mathrm{H}^{\mathbf{I}}, \mathrm{H}^{\mathrm{J}}\right)$.

4-\{4-(4-Methoxy-4'-oxybiphenyl)benzophenone $(\mathrm{R}=4$-methoxy-4'-oxybiphenyl, $m=1)$.

Recrystallized from THF. mp $199.6^{\circ} \mathrm{C}$. Yield $73.3 \%$.

Chemical shifts $\delta 1.78-2.22\left(\mathrm{~m}, 4 \mathrm{H}, \mathrm{H}^{\mathrm{E}}\right.$, $\left.\mathrm{H}^{\mathrm{F}}\right), 3.83\left(\mathrm{~s}, 3 \mathrm{H}, \mathrm{H}^{\mathrm{A}}\right), 4.18-4.32\left(\mathrm{~m}, 4 \mathrm{H}, \mathrm{H}^{\mathrm{D}}\right.$, $\left.\mathrm{H}^{\mathrm{G}}\right), \quad 6.94\left(\mathrm{~d}, 6 \mathrm{H}, \quad J=9.0 \mathrm{~Hz}, \mathrm{H}^{\mathrm{B}}, \mathrm{H}^{\mathrm{H}}\right)$, $7.30-7.65\left(\mathrm{~m}, 3 \mathrm{H}, \mathrm{H}^{\mathrm{K}}\right), 7.48(\mathrm{~d}, 4 \mathrm{H}, J=9.0 \mathrm{~Hz}$, $\left.\mathrm{H}^{\mathrm{c}}\right), 7.65-7.98\left(\mathrm{~m}, 4 \mathrm{H}, J=9.0 \mathrm{~Hz}, \mathrm{H}^{\mathrm{I}}, \mathrm{H}^{\mathrm{J}}\right)$.

4-\{5-(4-Methoxy-4'-oxybiphenyl)pentyloxy\}benzophenone ( $\mathrm{R}=4$-methoxy-4'-oxybiphenyl, $m=2$ ).

Recrystallized from THF. mp $158.3^{\circ} \mathrm{C}$. Yield $63.4 \%$.

Chemical shifts $\delta 1.26-2.12\left(\mathrm{~m}, 6 \mathrm{H}, \mathrm{H}^{\mathrm{E}}\right.$, $\left.\mathrm{H}^{\mathrm{F}}\right), 3.83\left(\mathrm{~s}, 3 \mathrm{H}, \mathrm{H}^{\mathrm{A}}\right), 3.86-4.25\left(\mathrm{~m}, 4 \mathrm{H}, \mathrm{H}^{\mathrm{D}}\right.$, $\left.\mathrm{H}^{\mathrm{G}}\right), \quad 6.94\left(\mathrm{~d}, \quad 6 \mathrm{H}, \quad J=9.0 \mathrm{~Hz}, \mathrm{H}^{\mathrm{B}}, \mathrm{H}^{\mathrm{H}}\right)$, $7.32-7.63\left(\mathrm{~m}, 3 \mathrm{H}, \mathrm{H}^{\mathrm{K}}\right), 7.47(\mathrm{~d}, 4 \mathrm{H}, J=9.0 \mathrm{~Hz}$, $\left.\mathrm{H}^{\mathrm{C}}\right), 7.65-7.95\left(\mathrm{~m}, 4 \mathrm{H}, J=9.0 \mathrm{~Hz}, \mathrm{H}^{\mathrm{I}}, \mathrm{H}^{\mathrm{J}}\right)$. 
phenyl)alkoxy\}phenyl]diphenylmethanol and purified by column chromatography. A typical example is given.

(4-Methoxyphenyl)diphenylmethanol. To the ether $(50 \mathrm{ml})$ solution of 4-methoxybenzophenone ( $5.38 \mathrm{~g}, 25.35 \mathrm{mmol})$ was added dropwise ether solution $(150 \mathrm{ml})$ of phenyllithium $(1 \mathrm{~N})$ during $1 \mathrm{~h}$. After stirring for $12 \mathrm{~h}$ at room temperature, the reaction mixture was decomposed by slowly adding diluted hydrochloric acid $(200 \mathrm{ml})$. The water layer was extracted twice with $100 \mathrm{ml}$ ether after separating the organic layer. Crude product was obtained by the evaporation of the solvent after drying the organic solution over magnesium sulfate. The crude product was purified by silica gel column chromatography using hexane-ether $(3: 1)$ as an eluent $(R f=0.20)$.

Yield $99.2 \% . \mathrm{mp} 65.8^{\circ} \mathrm{C}$.

Chemical shifts $\delta 2.78\left(\mathrm{~s}, 1 \mathrm{H}, \mathrm{H}^{\mathrm{L}}\right), 3.79(\mathrm{~s}$, $\left.3 \mathrm{H}, \mathrm{CH}_{3}\right), 6.83\left(\mathrm{~d}, 2 \mathrm{H}, J=9.0 \mathrm{~Hz}, \mathrm{H}^{\mathrm{H}}\right), 7.17$ $\left(\mathrm{d}, 2 \mathrm{H}, \mathrm{H}^{\mathrm{l}}\right), 7.29\left(\mathrm{~s}, 10 \mathrm{H}, \mathrm{H}^{\mathrm{J}}, \mathrm{H}^{\mathrm{K}}\right)$.

(4-Ethoxyphenyl)diphenylmethanol.

$R f=0.16$ (hexane-ether, $5: 1$ ). Yield quant.

Chemical shifts $\delta 1.43(\mathrm{t}, 3 \mathrm{H}, J=7.0 \mathrm{~Hz}$, $\left.\mathrm{CH}_{3}\right), 2.81\left(\mathrm{~s}, 1 \mathrm{H}, \mathrm{H}^{\mathrm{L}}\right), 4.04(\mathrm{q}, 2 \mathrm{H}, J=7.0 \mathrm{~Hz}$, $\left.\mathrm{OCH}_{2}\right), 6.85\left(\mathrm{~d}, 2 \mathrm{H}, J=9.0 \mathrm{~Hz}, \mathrm{H}^{\mathrm{H}}\right), 7.18(\mathrm{~d}$, $\left.2 \mathrm{H}, \mathrm{H}^{\mathrm{I}}\right), 7.27-7.37\left(\mathrm{~m}, 10 \mathrm{H}, \mathrm{H}^{\mathrm{J}}, \mathrm{H}^{\mathrm{K}}\right)$.

(4-Propoxyphenyl)diphenylmethanol $(\mathrm{R}=\mathrm{H}$, $m=0)$.

$R f=0.16$ (hexane-ether, $5: 1$ ). Yield 91.3\%.

Chemical shifts $\delta 1.00\left(\mathrm{t}, 3 \mathrm{H}, J=7.0 \mathrm{~Hz}, \mathrm{H}^{\mathrm{D}}\right)$, $1.78\left(\mathrm{q}, 2 \mathrm{H}, J=7.0 \mathrm{~Hz}, \mathrm{H}^{\mathrm{F}}\right), 2.72\left(\mathrm{~s}, 1 \mathrm{H}, \mathrm{H}^{\mathrm{L}}\right)$, $3.89\left(\mathrm{t}, 2 \mathrm{H}, J=7.0 \mathrm{~Hz}, \mathrm{H}^{\mathrm{G}}\right), 6.81(\mathrm{~d}, 2 \mathrm{H}$, $\left.J=9.0 \mathrm{~Hz}, \mathrm{H}^{\mathrm{H}}\right), 7.13\left(\mathrm{~d}, 2 \mathrm{H}, \mathrm{H}^{\mathrm{I}}\right), 7.22-7.37$ $\left(\mathrm{m}, 10 \mathrm{H}, \mathrm{H}^{\mathrm{J}}, \mathrm{H}^{\mathrm{K}}\right)$.

(4-Butoxyphenyl)diphenylmethanol $(\mathrm{R}=\mathrm{H}$, $m=1)$.

$R f=0.25$ (hexane-ether, $4: 1$ ). Yield quant. Chemical shifts $\delta 0.96\left(\mathrm{t}, 3 \mathrm{H}, J=7.0 \mathrm{~Hz}, \mathrm{H}^{\mathrm{D}}\right)$, $1.16-2.15\left(\mathrm{~m}, 4 \mathrm{H}, \mathrm{H}^{\mathrm{E}}, \mathrm{H}^{\mathrm{F}}\right), 2.71\left(\mathrm{~s}, 1 \mathrm{H}, \mathrm{H}^{\mathrm{L}}\right)$, $3.94\left(\mathrm{t}, 2 \mathrm{H}, J=7.0 \mathrm{~Hz}, \mathrm{H}^{\mathrm{G}}\right), 7.00(\mathrm{~d}, 2 \mathrm{H}$, $\left.J=9.0 \mathrm{~Hz}, \mathrm{H}^{\mathrm{H}}\right), 7.17\left(\mathrm{~d}, 2 \mathrm{H}, \mathrm{H}^{\mathrm{l}}\right), 7.27(\mathrm{~s}, 10 \mathrm{H}$, $\left.\mathrm{H}^{\mathrm{J}}, \mathrm{H}^{\mathrm{K}}\right)$.

(4-Pentyloxyphenyl)diphenylmethanol $\quad(\mathrm{R}=$ $\mathrm{H}, m=2)$.
$R f=0.16$ (hexane-ether, $5: 1$ ). Yield quant.

Chemical shifts $\delta 0.95(\mathrm{t}, 3 \mathrm{H}, J=7.0 \mathrm{~Hz}$, $\left.\mathrm{H}^{\mathrm{D}}\right), 1.36-1.51\left(\mathrm{~m}, 4 \mathrm{H}, \mathrm{H}^{\mathrm{E}}\right), 1.80$ (quint, $2 \mathrm{H}$, $\left.\mathrm{H}^{\mathrm{F}}\right), 2.79\left(\mathrm{~s}, 1 \mathrm{H}, \mathrm{H}^{\mathrm{L}}\right), 3.96(\mathrm{t}, 2 \mathrm{H}, J=7.0 \mathrm{~Hz}$, $\left.\mathrm{H}^{\mathrm{G}}\right), 6.97\left(\mathrm{~d}, 2 \mathrm{H}, J=9.0 \mathrm{~Hz}, \mathrm{H}^{\mathrm{H}}\right), 7.20(\mathrm{~d}, 2 \mathrm{H}$, $\left.\mathrm{H}^{\mathrm{l}}\right), 7.23-7.34\left(\mathrm{~m}, 10 \mathrm{H}, \mathrm{H}^{\mathrm{J}}, \mathrm{H}^{\mathrm{K}}\right)$.

(4-Hexyloxyphenyl)diphenylmethanol $\quad(\mathrm{R}=$ $\mathrm{H}, m=3)$.

$R f=0.16$ (hexane-ether, $5: 1$ ). Yield quant. Chemical shifts $\delta 0.88\left(\mathrm{t}, 3 \mathrm{H}, J=7.0 \mathrm{~Hz}, \mathrm{H}^{\mathrm{D}}\right)$, $1.20-1.55\left(\mathrm{~m}, 6 \mathrm{H}, \mathrm{H}^{\mathrm{E}}\right), 1.75$ (quint, $2 \mathrm{H}, \mathrm{H}^{\mathrm{F}}$ ), $2.73\left(\mathrm{~s}, 1 \mathrm{H}, \mathrm{H}^{\mathrm{L}}\right), 3.92\left(\mathrm{t}, 2 \mathrm{H}, J=7.0 \mathrm{~Hz}, \mathrm{H}^{\mathrm{G}}\right)$, $6.80\left(\mathrm{~d}, 2 \mathrm{H}, J=9.0 \mathrm{~Hz}, \mathrm{H}^{\mathrm{H}}\right), 7.13\left(\mathrm{~d}, 2 \mathrm{H}, \mathrm{H}^{\mathrm{I}}\right)$, $7.22-7.34\left(\mathrm{~m}, 10 \mathrm{H}, \mathrm{H}^{\mathrm{J}}, \mathrm{H}^{\mathrm{K}}\right)$.

(4-Heptyloxyphenyl)diphenylmethanol $(\mathrm{R}=$ $\mathrm{H}, m=4)$.

$R f=0.17$ (hexane-ether, $5: 1$ ). Yield quant.

Chemical shifts $\delta 0.87\left(\mathrm{t}, 3 \mathrm{H}, J=7.0 \mathrm{~Hz}, \mathrm{H}^{\mathrm{D}}\right)$, $1.18-1.54\left(\mathrm{~m}, 8 \mathrm{H}, \mathrm{H}^{\mathrm{E}}\right), 1.75$ (quint, $2 \mathrm{H}, \mathrm{H}^{\mathrm{F}}$ ), $2.73\left(\mathrm{~s}, 1 \mathrm{H}, \mathrm{H}^{\mathrm{L}}\right), 3.92\left(\mathrm{t}, 2 \mathrm{H}, J=7.0 \mathrm{~Hz}, \mathrm{H}^{\mathrm{G}}\right)$, $6.81\left(\mathrm{~d}, 2 \mathrm{H}, J=9.0 \mathrm{~Hz}, \mathrm{H}^{\mathrm{H}}\right), 7.13\left(\mathrm{~d}, 2 \mathrm{H}, \mathrm{H}^{\mathrm{I}}\right)$, $7.22-7.35\left(\mathrm{~m}, 10 \mathrm{H}, \mathrm{H}^{\mathrm{J}}, \mathrm{H}^{\mathrm{K}}\right)$.

(4-Octyloxyphenyl)diphenylmethanol $(\mathrm{R}=\mathrm{H}$, $m=5)$.

$R f=0.29$ (hexane-ether, $4: 1)$. Yield quant.

Chemical shifts $\delta 0.94\left(\mathrm{t}, 3 \mathrm{H}, J=7.0 \mathrm{~Hz}, \mathrm{H}^{\mathrm{D}}\right)$, $1.13-2.03\left(\mathrm{~m}, 12 \mathrm{H}, \mathrm{H}^{\mathrm{E}}, \mathrm{H}^{\mathrm{F}}\right), 2.80\left(\mathrm{~s}, 1 \mathrm{H}, \mathrm{H}^{\mathrm{L}}\right)$, $4.00\left(\mathrm{t}, 2 \mathrm{H}, J=7.0 \mathrm{~Hz}, \mathrm{H}^{\mathrm{G}}\right), 6.86(\mathrm{~d}, 2 \mathrm{H}$, $\left.J=9.0 \mathrm{~Hz}, \mathrm{H}^{\mathrm{H}}\right), 7.13\left(\mathrm{~d}, 2 \mathrm{H}, \mathrm{H}^{\mathrm{I}}\right), 7.32(\mathrm{~s}, 10 \mathrm{H}$, $\left.\mathrm{H}^{\mathrm{J}}, \mathrm{H}^{\mathrm{K}}\right)$.

[4-\{3-(4-Methoxy-4'-oxybiphenyl)propo$x y\}$ phenyl]diphenylmethanol $(\mathrm{R}=4$-methoxy4'-oxybiphenyl, $m=0$ ).

$R f=0.21$ (hexane-ether, 2:1). Yield 91.6\%. $\mathrm{mp} 49.7^{\circ} \mathrm{C}$.

Chemical shifts $\delta 2.27(\mathrm{dt}, 2 \mathrm{H}, J=6.0 \mathrm{~Hz}$, $\left.\mathrm{H}^{\mathrm{F}}\right), 2.73\left(\mathrm{~s}, 1 \mathrm{H}, \mathrm{H}^{\mathrm{L}}\right), 3.84\left(\mathrm{~s}, 3 \mathrm{H}, \mathrm{H}^{\mathrm{A}}\right), 4.19(\mathrm{t}$, $4 \mathrm{H}, J=6.0 \mathrm{~Hz}, \mathrm{H}^{\mathrm{D}}, \mathrm{H}^{\mathrm{G}}$ ), 6.85, 6.93 (two, d, $6 \mathrm{H}$, $\left.J=9.0 \mathrm{~Hz}, \mathrm{H}^{\mathrm{B}}, \mathrm{H}^{\mathrm{H}}\right), 7.20(\mathrm{~d}, 2 \mathrm{H}, J=9.0 \mathrm{~Hz}$, $\left.\mathrm{H}^{\mathrm{l}}\right), 7.29\left(\mathrm{~s}, 10 \mathrm{H}, \mathrm{H}^{\mathrm{J}}, \mathrm{H}^{\mathrm{K}}\right), 7.48(\mathrm{~d}, 4 \mathrm{H}$, $\left.J=9.0 \mathrm{~Hz}, \mathrm{H}^{\mathrm{C}}\right)$.

[4-\{4-(4-Methoxy-4'-oxybiphenyl)butoxy\}phenyl]diphenylmethanol $(\mathrm{R}=4$-methoxy-4'oxybiphenyl, $m=1$ ).

Recrystallized from ethanol. Yield $87.5 \%$. $\mathrm{mp} 123.6^{\circ} \mathrm{C}$. 
Chemical shifts $\delta 1.75-2.20\left(\mathrm{~m}, 4 \mathrm{H}, \mathrm{H}^{\mathrm{E}}\right.$, $\left.\mathrm{H}^{\mathrm{F}}\right), 2.71\left(\mathrm{~s}, 1 \mathrm{H}, \mathrm{H}^{\mathrm{L}}\right), 3.84\left(\mathrm{~s}, 3 \mathrm{H}, \mathrm{H}^{\mathrm{A}}\right)$, $3.90-4.23\left(\mathrm{~m}, 4 \mathrm{H}, \mathrm{H}^{\mathrm{D}}, \mathrm{H}^{\mathrm{G}}\right), 6.836 .93$ (two d, $\left.6 \mathrm{H}, J=9.0 \mathrm{~Hz}, \mathrm{H}^{\mathrm{B}}, \mathrm{H}^{\mathrm{H}}\right), 7.18(\mathrm{~d}, 2 \mathrm{H}, J=9.0 \mathrm{~Hz}$, $\left.\mathrm{H}^{\mathrm{I}}\right), 7.29\left(\mathrm{~s}, 10 \mathrm{H}, \mathrm{H}^{\mathrm{J}}, \mathrm{H}^{\mathrm{K}}\right), 7.47(\mathrm{~d}, 4 \mathrm{H}$, $\left.J=9.0 \mathrm{~Hz}, \mathrm{H}^{\mathrm{C}}\right)$.

[4-\{5-(4-Methoxy-4'-oxybiphenyl)pentylo$x y\}$ phenyl]diphenylmethanol $(\mathrm{R}=4$-methoxy4'-oxybiphenyl, $m=2$ ).
Recrystallized from methanol. Yield 57.5\%. $\mathrm{mp} 49.2^{\circ} \mathrm{C}$.

Chemical shifts $\delta 1.60-2.10\left(\mathrm{~m}, 6 \mathrm{H}, \mathrm{H}^{\mathrm{E}}\right.$, $\left.\mathrm{H}^{\mathrm{F}}\right), 2.71\left(\mathrm{~s}, 1 \mathrm{H}, \mathrm{H}^{\mathrm{L}}\right), 3.84\left(\mathrm{~s}, 3 \mathrm{H}, \mathrm{H}^{\mathrm{A}}\right)$, $3.83-4.20\left(\mathrm{~m}, 4 \mathrm{H}, \mathrm{H}^{\mathrm{D}}, \mathrm{H}^{\mathrm{G}}\right), 6.82,6.94$ (two d, $\left.6 \mathrm{H}, J=9.0 \mathrm{~Hz}, \mathrm{H}^{\mathrm{B}}, \mathrm{H}^{\mathrm{H}}\right), 7.18(\mathrm{~d}, 2 \mathrm{H}, J=9.0 \mathrm{~Hz}$, $\left.\mathrm{H}^{\mathrm{I}}\right), 7.29\left(\mathrm{~s}, 10 \mathrm{H}, \mathrm{H}^{\mathrm{J}}, \mathrm{H}^{\mathrm{K}}\right), 7.47(\mathrm{~d}, 4 \mathrm{H}$, $J=9.0 \mathrm{~Hz}, \mathrm{H}^{\mathrm{C}}$ ).

Trimethylsiloxytriphenylmethanes

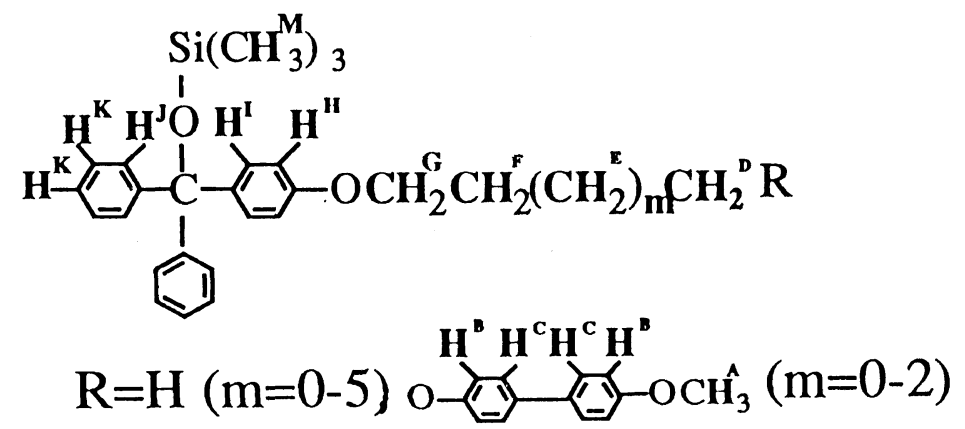

The substituted triphenylmethanols synthesized above were converted into trimethylsilyl ethers by the reaction with trimethylsilyl chloride. A typical example is given.

Trimethylsiloxytriphenylmethane. To the mixture of triphenylmethanol $(1.00 \mathrm{~g}, 3.84$ $\mathrm{mmol})$ and sodium hydride $(0.24 \mathrm{~g}, 10.0 \mathrm{mmol})$ in a flask, dimethylformamide $(70 \mathrm{ml})$ was added and the reaction system was stirred for $5 \mathrm{~h}$ at $50^{\circ} \mathrm{C}$. After ' $5 \mathrm{~h}$, trimethylchlorosilane $(1.72 \mathrm{~g}, 15.8 \mathrm{mmol})$ was added to the reaction system, and the whole system was stirred for further $12 \mathrm{~h}$ at $60^{\circ} \mathrm{C}$. After $12 \mathrm{~h}$, excess trimethylchlorosilane and dimethylformamide were removed under vacuum, and the residual material was extracted with hexane. The hexane soluble fraction was concentrated and purified by column chromatography.

$R f=0.17$ (hexane). Yield quant. $\mathrm{mp} 34.9^{\circ} \mathrm{C}$.

Chemical shifts $\delta-0.13\left(\mathrm{~s}, 9 \mathrm{H}, \mathrm{H}^{\mathrm{M}}\right), 7.04$ (m, 15H, $\left.\mathrm{H}^{\mathrm{J}}, \mathrm{H}^{\mathrm{K}}\right)$.

Other trimethylsiloxy substituted triphenylmethanes are liquid unless otherwise noted.

Trimethylsiloxy(4-methoxyphenyl)diphenylmethane. $R f=0.28$ (hexane-ether, 49:1).
Yield 88.1\%.

Chemical shifts $\delta-0.17\left(\mathrm{~s}, 9 \mathrm{H}, \mathrm{H}^{\mathrm{M}}\right), 3.78$ (s, 3H, $\left.\mathrm{CH}_{3}\right), 6.80\left(\mathrm{~d}, 2 \mathrm{H}, J=9.0 \mathrm{~Hz}, \mathrm{H}^{\mathrm{H}}\right), 7.24$ $\left(\mathrm{d}, 2 \mathrm{H}, \mathrm{H}^{\mathrm{I}}\right), 7.17-7.46\left(\mathrm{~m}, 10 \mathrm{H}, \mathrm{H}^{\mathrm{J}}, \mathrm{H}^{\mathrm{K}}\right)$.

Trimethylsiloxy(4-ethoxyphenyl)diphenylmethane.

$R f=0.30$ (hexane-ether, $40: 1$ ). Yield $84.9 \%$.

Chemical shifts $\delta-0.17\left(\mathrm{~s}, 9 \mathrm{H}, \mathrm{H}^{\mathrm{M}}\right), 1.38$ (t, $\left.3 \mathrm{H}, J=7.0 \mathrm{~Hz}, \mathrm{CH}_{3}\right), 4.00\left(\mathrm{q}, 2 \mathrm{H}, \mathrm{OC}_{2}\right)$, $6.79\left(\mathrm{~d}, 2 \mathrm{H}, J=9.0 \mathrm{~Hz}, \mathrm{H}^{\mathrm{H}}\right), 7.14-7.45(\mathrm{~m}$, $\left.12 \mathrm{H}, \mathbf{H}^{\mathbf{l}}, \mathbf{H}^{\mathbf{J}}, \mathbf{H}^{\mathbf{K}}\right)$.

Trimethylsiloxy(4-propoxyphenyl)diphenylmethane $(\mathrm{R}=\mathrm{H}, m=0)$.

$R f=0.30$ (hexane-ether, $40: 1$ ). Yield $88.8 \%$.

Chemical shifts $\delta-0.17\left(\mathrm{~s}, 9 \mathrm{H}, \mathrm{H}^{\mathrm{M}}\right), 1.01$ $\left(\mathrm{t}, 3 \mathrm{H}, J=7.0 \mathrm{~Hz}, \mathrm{H}^{\mathrm{D}}\right), 1.78\left(\mathrm{~m}, 2 \mathrm{H}, \mathrm{H}^{\mathrm{F}}\right), 3.89$ $\left(\mathrm{t}, 2 \mathrm{H}, J=7.0 \mathrm{~Hz}, \mathrm{H}^{\mathrm{G}}\right), 6.79(\mathrm{~d}, 2 \mathrm{H}, J=9.0 \mathrm{~Hz}$, $\left.\mathrm{H}^{\mathrm{H}}\right), 7.13-7.47\left(\mathrm{~m}, 12 \mathrm{H}, \mathrm{H}^{\mathrm{I}}, \mathrm{H}^{\mathrm{J}}, \mathrm{H}^{\mathrm{K}}\right)$.

Trimethylsiloxy(4-butoxyphenyl)diphenylmethane $(\mathrm{R}=\mathrm{H}, m=1)$.

$R f=0.32$ (hexane-ether, $40: 1$ ). Yield quant.

Chemical shifts $\delta-0.17\left(\mathrm{~s}, 9 \mathrm{H}, \mathrm{H}^{\mathrm{M}}\right), 0.97$ $\left(\mathrm{t}, 3 \mathrm{H}, J=7.0 \mathrm{~Hz}, \mathrm{H}^{\mathrm{D}}\right), 1.15-2.15\left(\mathrm{~m}, 4 \mathrm{H}, \mathrm{H}^{\mathrm{E}}\right.$, $\left.\mathrm{H}^{\mathrm{F}}\right), 3.95\left(\mathrm{t}, 2 \mathrm{H}, J=7.0 \mathrm{~Hz}, \mathrm{H}^{\mathrm{G}}\right), 6.79(\mathrm{~d}, 2 \mathrm{H}$, $\left.J=9.0 \mathrm{~Hz}, \mathrm{H}^{\mathrm{H}}\right), 7.17-7.50\left(\mathrm{~m}, 12 \mathrm{H}, \mathrm{H}^{\mathrm{I}}, \mathrm{H}^{\mathrm{J}}\right.$, 
$\left.\mathbf{H}^{\mathrm{K}}\right)$.

Trimethylsiloxy(4-pentyloxyphenyl)diphenylmethane $(\mathrm{R}=\mathrm{H}, m=2)$.

$R f=0.32$ (hexane-ether, $40: 1$ ). Yield $83.8 \%$.

Chemical shifts $\delta-0.17\left(\mathrm{~s}, 9 \mathrm{H}, \mathrm{H}^{\mathrm{M}}\right), 0.91$ $\left(\mathrm{t}, 3 \mathrm{H}, J=7.0 \mathrm{~Hz}, \mathrm{H}^{\mathrm{D}}\right), 1.20-1.52\left(\mathrm{~m}, 4 \mathrm{H}, \mathrm{H}^{\mathrm{E}}\right)$, 1.76 (quint, $\left.2 \mathrm{H}, J=7.0 \mathrm{~Hz}, \mathrm{H}^{\mathrm{F}}\right), 3.92(\mathrm{t}, 2 \mathrm{H}$, $\left.J=7.0 \mathrm{~Hz}, \mathrm{H}^{\mathrm{G}}\right), 6.78\left(\mathrm{~d}, 2 \mathrm{H}, J=9.0 \mathrm{~Hz}, \mathrm{H}^{\mathrm{H}}\right)$, $7.13-7.48\left(\mathrm{~m}, 12 \mathrm{H}, \mathrm{H}^{\mathrm{I}}, \mathrm{H}^{\mathrm{J}}, \mathrm{H}^{\mathrm{K}}\right)$.

Trimethylsiloxy(4-hexyloxyphenyl)diphenylmethane $(\mathrm{R}=\mathrm{G}, m=3)$.

$R f=0.32$ (hexane-ether, $40: 1$ ). Yield $82.6 \%$.

Chemical shifts $\delta-0.17\left(\mathrm{~s}, 9 \mathrm{H}, \mathrm{H}^{\mathrm{M}}\right), 0.88$ $\left(\mathrm{t}, 3 \mathrm{H}, J=7.0 \mathrm{~Hz}, \mathrm{H}^{\mathrm{D}}\right), 1.20-1.52\left(\mathrm{~m}, 6 \mathrm{H}, \mathrm{H}^{\mathrm{E}}\right)$, 1.75 (quint, $2 \mathrm{H}, J=7.0 \mathrm{~Hz}, \mathrm{H}^{\mathrm{F}}$ ), $3.92(\mathrm{t}, 2 \mathrm{H}$, $\left.J=7.0 \mathrm{~Hz}, \mathrm{H}^{\mathrm{G}}\right), 6.78\left(\mathrm{~d}, 2 \mathrm{H}, J=9.0 \mathrm{~Hz}, \mathrm{H}^{\mathrm{H}}\right)$, 7.13-7.47 (m, 12H, $\left.\mathrm{H}^{\mathrm{I}}, \mathrm{H}^{\mathrm{J}}, \mathrm{H}^{\mathrm{K}}\right)$.

Trimethylsiloxy(4-heptyloxyphenyl)diphenylmethane $(\mathrm{R}=\mathrm{H}, m=4)$.

$R f=0.32$ (hexane-ether, $40: 1$ ). Yield $65.8 \%$.

Chemical shifts $\delta-0.17\left(\mathrm{~s}, 9 \mathrm{H}, \mathrm{H}^{\mathrm{M}}\right), 0.87$ $\left(\mathrm{t}, 3 \mathrm{H}, J=7.0 \mathrm{~Hz}, \mathrm{H}^{\mathrm{D}}\right), 1.18-1.52\left(\mathrm{~m}, 8 \mathrm{H}, \mathrm{H}^{\mathrm{E}}\right)$, 1.75 (quint, $2 \mathrm{H}, J=7.0 \mathrm{~Hz}, \mathrm{H}^{\mathrm{F}}$ ), $3.92(\mathrm{t}, 2 \mathrm{H}$, $\left.J=7.0 \mathrm{~Hz}, \mathrm{H}^{\mathrm{G}}\right), 6.78\left(\mathrm{~d}, 2 \mathrm{H}, J=9.0 \mathrm{~Hz}, \mathrm{H}^{\mathrm{H}}\right)$, 7.14-7.47 (m, 12H, $\left.\mathbf{H}^{\mathbf{I}}, \mathbf{H}^{\mathrm{J}}, \mathbf{H}^{\mathrm{K}}\right)$.

Trimethylsiloxy(4-octyloxyphenyl)diphenylmethane $(\mathrm{R}=\mathrm{H}, m=5)$.

$R f=0.32$ (hexane-ether, $40: 1$ ). Yield $98.0 \%$.

Chemical shifts $\delta-0.17\left(\mathrm{~s}, 9 \mathrm{H}, \mathrm{H}^{\mathrm{M}}\right), 0.94$ $\left(\mathrm{t}, 3 \mathrm{H}, J=7.0 \mathrm{~Hz}, \mathrm{H}^{\mathrm{D}}\right), 1.10-2.00(\mathrm{~m}, 12 \mathrm{H}$, $\left.\mathrm{H}^{\mathrm{E}}, \mathrm{H}^{\mathrm{F}}\right), 3.99\left(\mathrm{t}, 2 \mathrm{H}, J=7.0 \mathrm{~Hz}, \mathrm{H}^{\mathrm{G}}\right), 6.85(\mathrm{~d}$, $\left.2 \mathrm{H}, J=9.0 \mathrm{~Hz}, \mathrm{H}^{\mathrm{H}}\right), 7.09-7.62\left(\mathrm{~m}, 12 \mathrm{H}, \mathrm{H}^{\mathrm{I}}\right.$,

Triphenylmethyl methacrylates

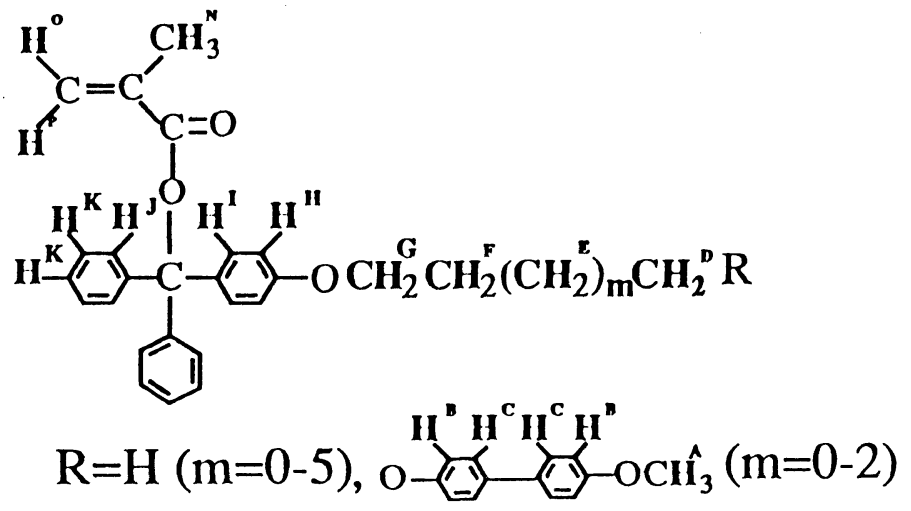

$\left.\mathbf{H}^{\mathrm{J}}, \mathrm{H}^{\mathrm{K}}\right)$

Trimethylsiloxy [4-\{3-(4-Methoxy-4'-oxybiphenyl)propoxy\}phenyl]diphenylmethane $(\mathrm{R}=$ 4-methoxy-4'-oxybiphenyl, $m=0$ ).

$R f=0.32$ (hexane-ether, $6: 1$ ). Yield $97.8 \%$. $\mathrm{mp} 100^{\circ} \mathrm{C}$.

Chemical shifts $\delta-0.17\left(\mathrm{~s}, 9 \mathrm{H}, \mathrm{H}^{\mathrm{M}}\right), 2.27$ $\left(\mathrm{dt}, 2 \mathrm{H}, J=7.0 \mathrm{~Hz}, \mathrm{H}^{\mathrm{F}}\right), 3.84\left(\mathrm{~s}, 3 \mathrm{H}, \mathrm{H}^{\mathrm{A}}\right), 4.07$ (t, $4 \mathrm{H}, J=7.0 \mathrm{~Hz}, \mathrm{H}^{\mathrm{D}}, \mathrm{H}^{\mathrm{G}}$ ), 6.83, 6.96 (two d, $\left.6 \mathrm{H}, J=9.0 \mathrm{~Hz}, \mathrm{H}^{\mathrm{B}}, \mathrm{H}^{\mathrm{H}}\right), 7.11-7.66(\mathrm{~m}, 12 \mathrm{H}$, $\left.\mathrm{H}^{\mathrm{I}}, \mathrm{H}^{\mathrm{J}}, \mathrm{H}^{\mathrm{K}}\right), 7.48\left(\mathrm{~d}, 4 \mathrm{H}, J=9.0 \mathrm{~Hz}, \mathrm{H}^{\mathrm{C}}\right)$.

Trimethylsiloxy [4-\{4-(4-Methoxy-4'-oxybiphenyl)butoxy $\}$ phenyl]diphenylmethane $(\mathrm{R}=4$ methoxy-4'-oxybiphenyl, $m=1$ ).

$R f=0.32$ (hexane-ether, $6: 1$ ). Yield $95.5 \%$. $\mathrm{mp} 43.0^{\circ} \mathrm{C}$.

Chemical shifts $\delta-0.17\left(\mathrm{~s}, 9 \mathrm{H}, \mathrm{H}^{\mathrm{M}}\right)$, $1.76-2.18\left(\mathrm{~m}, 4 \mathrm{H}, \mathrm{H}^{\mathrm{E}}, \mathrm{H}^{\mathrm{F}}\right), 3.84\left(\mathrm{~s}, 3 \mathrm{H}, \mathrm{H}^{\mathrm{A}}\right)$, $3.86-4.26\left(\mathrm{~m}, 4 \mathrm{H}, \mathrm{H}^{\mathrm{D}}, \mathrm{H}^{\mathrm{G}}\right), 6.81,6.95$ (two d, $\left.6 \mathrm{H}, J=9.0 \mathrm{~Hz}, \mathrm{H}^{\mathrm{B}}, \mathrm{H}^{\mathrm{H}}\right), 7.14-7.63(\mathrm{~m}, 12 \mathrm{H}$, $\left.\mathrm{H}^{\mathrm{I}}, \mathrm{H}^{\mathrm{J}}, \mathrm{H}^{\mathrm{K}}\right), 7.48\left(\mathrm{~d}, 4 \mathrm{H}, J=9.0 \mathrm{~Hz}, \mathrm{H}^{\mathrm{C}}\right)$.

Trimethylsiloxy [4-\{5-(4-Methoxy-4'-oxybiphenyl)pentyloxy\}phenyl]diphenylmethane ( $R=4$-methoxy-4'-oxybiphenyl, $m=2$ ).

$R f=0.32$ (hexane-ether, $6: 1$ ). Yield $97.9 \%$, $\mathrm{mp} 39.2^{\circ} \mathrm{C}$.

Chemical shifts $\delta-0.17\left(\mathrm{~s}, 9 \mathrm{H}, \mathrm{H}^{\mathrm{M}}\right)$, $1.55-2.08\left(\mathrm{~m}, 6 \mathrm{H}, \mathrm{H}^{\mathrm{E}}, \mathrm{H}^{\mathrm{F}}\right), 3.84\left(\mathrm{~s}, 3 \mathrm{H}, \mathrm{H}^{\mathrm{A}}\right)$, $3.84-4.17\left(\mathrm{~m}, 4 \mathrm{H}, \mathrm{H}^{\mathrm{D}}, \mathrm{H}^{\mathrm{G}}\right), 6.82,6.95$ (two d, $\left.6 \mathrm{H}, J=9.0 \mathrm{~Hz}, \mathrm{H}^{\mathrm{B}}, \mathrm{H}^{\mathrm{H}}\right), 7.15-7.50(\mathrm{~m}, 12 \mathrm{H}$, $\left.\mathrm{H}^{\mathrm{I}}, \mathrm{H}^{\mathrm{J}}, \mathrm{H}^{\mathrm{K}}\right), 7.48\left(\mathrm{~d}, 4 \mathrm{H}, J=9.0 \mathrm{~Hz}, \mathrm{H}^{\mathrm{c}}\right)$. 
Trimethylsiloxytriphenylmethane was converted into methacrylate by the reaction with trimethylsilyl methacrylate in the presence of trimethylsilyl trifluoromethanesulfonate as a catalyst. A typical example is given.

Triphenylmethyl Methacrylate (TrMA). Reaction of trimethylsilyl methacrylate (1.79 $\mathrm{g}, 11.30 \mathrm{mmol})$ and trimethylsiloxytriphenylmethane $(3.75 \mathrm{~g}, 28 \mathrm{mmol})$ was carried out in dichloromethane $(56 \mathrm{ml})$ for $12 \mathrm{~h}$ at $0^{\circ} \mathrm{C}$ in the presence of catalytic amounts of trimethylsilyl trifluoromethanesulfonate $(26 \mathrm{mg}$, $0.12 \mathrm{mmol}, 1 \mathrm{~mol} \%$ ). After $12 \mathrm{~h}$, the reaction was stopped by the addition of pyridine $(0.1 \mathrm{ml})$ and washed with ice-cooled saturated aqueous sodium bicarbonate solution $(60 \mathrm{ml})$. Organic layer was separated and dried over potassium carbonate. After the evaporation of the solvent, hot hexane was added to the residual material, and hexane insoluble material was removed by filtration. Crude product obtained by the evaporation of hexane was recrystallized from hexane.

Yield 76.8\%. mp 97.7 ${ }^{\circ} \mathrm{C}$ (lit. 98-99 ${ }^{\circ} \mathrm{C}$ ).

Chemical shift $\delta 1.99$ (m with fine coupling, $3 \mathrm{H}, \mathrm{H}^{\mathrm{N}}$ ), $5.62\left(\mathrm{~m}\right.$ with fine coupling, $1 \mathrm{H}, \mathrm{H}^{\mathrm{O}}$ ), $6.24\left(\mathrm{~m}\right.$ with fine coupling, $1 \mathrm{H}, \mathrm{H}^{\mathrm{P}}$ ), 7.18-7.43 (m, 15H, phenyl protons).

In the synthesis of other monomers, the reaction products were purified on short path silica gel column chromatography at $0^{\circ} \mathrm{C}$ by directly loading the reaction mixture without pretreatments after stopping the reaction with pyridine.

(4-Methylphenyl)diphenylmethyl Methacrylate $(\mathrm{Me})$.

$R f=0.33$ (hexane-ether, 3:1). Oil. Yield $88.0 \%$.

Chemical shift $\delta 1.99$ ( $\mathrm{m}$ with fine coupling, $3 \mathrm{H}, \mathrm{H}^{\mathrm{N}}$ ), $2.32\left(\mathrm{~s}, 3 \mathrm{H}, \mathrm{C}_{3}\right), 5.61$ (m with fine coupling, $\left.1 \mathrm{H}, \mathrm{H}^{\mathrm{O}}\right), 6.24(\mathrm{~m}$ with fine coupling, $\left.1 \mathrm{H}, \quad \mathrm{H}^{\mathrm{P}}\right), \quad 7.10 \quad\left(\mathrm{~d}, \quad 2 \mathrm{H}, \quad J=9.0 \mathrm{~Hz}, \quad \mathrm{H}^{\mathrm{H}}\right)$, $7.22-7.43\left(\mathrm{~m}, 12 \mathrm{H}, \mathrm{H}^{\mathrm{I}}, \mathrm{H}^{\mathrm{J}}, \mathrm{H}^{\mathrm{K}}\right)$.

(4-Methoxyphenyl)diphenylmethyl Methacrylate $(\mathrm{MeO})$.

$R f=0.36$ (hexane-ether, 2:1). Oil. Yield
$89.3 \%$.

Chemical shift $\delta 1.99$ (m with fine coupling, $\left.3 \mathrm{H}, \mathrm{H}^{\mathrm{N}}\right), 3.78\left(\mathrm{~s}, 3 \mathrm{H}, \mathrm{OCH}_{3}\right), 5.60$ (m with fine coupling, $1 \mathrm{H}, \mathrm{H}^{\mathrm{O}}$ ), 6.23 ( $\mathrm{m}$ with fine coupling, $\left.1 \mathrm{H}, \mathrm{H}^{\mathrm{P}}\right), 6.82\left(\mathrm{~d}, 2 \mathrm{H}, J=9.0 \mathrm{~Hz}, \mathrm{H}^{\mathrm{H}}\right), 7.18(\mathrm{~d}$, $\left.2 \mathrm{H}, J=9.0 \mathrm{~Hz}, \mathrm{H}^{\mathrm{I}}\right), 7.21-7.42\left(\mathrm{~m}, 10 \mathrm{H}, \mathrm{H}^{\mathrm{J}}\right.$, $\mathrm{H}^{\mathrm{K}}$ ).

(4-Ethoxyphenyl)diphenylmethyl Methacrylate (EtO).

$R f=0.31$ (hexane-ether, 2:1). Oil. Yield $60.2 \%$.

Chemical shift $\delta 1.41(\mathrm{t}, 3 \mathrm{H}, J=7.0 \mathrm{~Hz}$, $\mathrm{CH}_{3}$ ), 2.00 ( $\mathrm{m}$ with fine coupling, $3 \mathrm{H}, \mathrm{H}^{\mathrm{N}}$ ), 4.03 (q, $2 \mathrm{H}, \mathrm{OCH}_{2}$ ), 5.62 ( $\mathrm{m}$ with fine coupling, $1 \mathrm{H}$, $\mathrm{H}^{\mathrm{O}}$ ), 6.24 ( $\mathrm{m}$ with fine coupling, $1 \mathrm{H}, \mathrm{H}^{\mathrm{P}}$ ), 6.82 $\left(\mathrm{d}, 2 \mathrm{H}, J=9.0 \mathrm{~Hz}, \mathrm{H}^{\mathrm{H}}\right), 7.17(\mathrm{~d}, 2 \mathrm{H}, J=9.0 \mathrm{~Hz}$, $\left.\mathrm{H}^{\mathrm{I}}\right), 7.20-7.44\left(\mathrm{~m}, 10 \mathrm{H}, \mathrm{H}^{\mathrm{J}}, \mathbf{H}^{\mathrm{K}}\right)$.

(4-Propoxyphenyl)diphenylmethyl Methacrylate $(\mathrm{R}=\mathrm{H}, m=0)(\mathrm{PrO})$.

$R f=0.20$ (hexane-ether, 4:1). Oil. Yield $68.4 \%$.

Chemical shift $\delta 1.00\left(\mathrm{t}, 3 \mathrm{H}, J=7.0 \mathrm{~Hz}, \mathrm{H}^{\mathrm{D}}\right)$, $1.77\left(\mathrm{~m}, 2 \mathrm{H}, \mathrm{H}^{\mathrm{F}}\right), 1.97(\mathrm{~m}$ with fine coupling, $\left.3 \mathrm{H}, \mathrm{H}^{\mathrm{N}}\right), 3.88\left(\mathrm{t}, 2 \mathrm{H}, \mathrm{H}^{\mathrm{G}}\right), 5.59(\mathrm{~m}$ with fine coupling, $1 \mathrm{H}, \mathrm{H}^{\mathrm{O}}$ ), 6.22 ( $\mathrm{m}$ with fine coupling, $\left.1 \mathrm{H}, \mathrm{H}^{\mathrm{P}}\right), 6.79\left(\mathrm{~d}, 2 \mathrm{H}, J=9.0 \mathrm{~Hz}, \mathrm{H}^{\mathrm{H}}\right), 7.12(\mathrm{~d}$, $\left.2 \mathrm{H}, J=9.0 \mathrm{~Hz}, \mathrm{H}^{\mathrm{I}}\right), 7.18-7.42\left(\mathrm{~m}, 10 \mathrm{H}, \mathrm{H}^{\mathrm{J}}\right.$, $\left.\mathrm{H}^{\mathrm{K}}\right)$.

(4-Butoxyphenyl)diphenylmethyl Methacrylate $(\mathrm{R}=\mathrm{H}, m=1)(\mathrm{BuO})$.

$R f=0.23$ (hexane-ether, 4:1). Oil. Yield $86.8 \%$.

Chemical shift $\delta 0.96\left(\mathrm{t}, 3 \mathrm{H}, J=7.0 \mathrm{~Hz}, \mathrm{H}^{\mathrm{D}}\right)$, $1.47-1.73\left(\mathrm{~m}, 4 \mathrm{H}, \mathrm{H}^{\mathrm{E}}, \mathrm{H}^{\mathrm{F}}\right), 1.98(\mathrm{~m}$ with fine coupling, $\left.3 \mathrm{H}, \mathrm{H}^{\mathrm{N}}\right), 3.94\left(\mathrm{t}, 2 \mathrm{H}, \mathrm{H}^{\mathrm{G}}\right), 5.59(\mathrm{~m}$ with fine coupling, $1 \mathrm{H}, \mathrm{H}^{\mathrm{O}}$ ), 6.23 (m with fine coupling, $\left.1 \mathrm{H}, \mathrm{H}^{\mathrm{P}}\right), 6.82\left(\mathrm{~d}, 2 \mathrm{H}, J=9.0 \mathrm{~Hz}, \mathrm{H}^{\mathrm{H}}\right)$, 7.12-7.42 (m, 12H, $\left.\mathrm{H}^{\mathrm{I}}, \mathrm{H}^{\mathrm{J}}, \mathrm{H}^{\mathrm{K}}\right)$.

(4-Pentyloxyphenyl)diphenylmethyl Methacrylate $(\mathrm{R}=\mathrm{H}, m=2)(\mathrm{PeO})$.

$R f=0.25$ (hexane-ether, $4: 1$ ). Oil. Yield $60.3 \%$.

Chemical shift $\delta 0.92\left(\mathrm{t}, 3 \mathrm{H}, J=7.0 \mathrm{~Hz}, \mathrm{H}^{\mathrm{D}}\right)$, $1.21-1.55\left(\mathrm{~m}, 4 \mathrm{H}, \mathrm{H}^{\mathrm{E}}\right), 1.77$ (quint, $2 \mathrm{H}, \mathrm{H}^{\mathrm{F}}$ ), $1.99\left(\mathrm{~m}\right.$ with fine coupling, $\left.3 \mathrm{H}, \mathrm{H}^{\mathrm{N}}\right), 3.92(\mathrm{t}$, $\left.2 \mathrm{H}, \mathrm{H}^{\mathrm{G}}\right), 5.61\left(\mathrm{~m}\right.$ with fine coupling, $1 \mathrm{H}, \mathrm{H}^{\mathrm{O}}$ ), 
$6.23\left(\mathrm{~m}\right.$ with fine coupling, $\left.1 \mathrm{H}, \mathrm{H}^{\mathrm{P}}\right), 6.81(\mathrm{~d}$, $\left.2 \mathrm{H}, J=9.0 \mathrm{~Hz}, \mathrm{H}^{\mathrm{H}}\right), 7.20-7.46\left(\mathrm{~m}, 12 \mathrm{H}, \mathrm{H}^{\mathrm{I}}\right.$, $\left.\mathrm{H}^{\mathrm{J}}, \mathbf{H}^{\mathrm{K}}\right)$.

(4-Hexyloxyphenyl)diphenylmethyl Methacrylate $(\mathrm{R}=\mathrm{H}, m=3)(\mathrm{HxO})$.

$R f=0.25$ (hexane-ether, $4: 1$ ). Oil. Yield $52.4 \%$.

Chemical shift $\delta 0.88\left(\mathrm{t}, 3 \mathrm{H}, J=7.0 \mathrm{~Hz}, \mathrm{H}^{\mathrm{D}}\right)$, $1.19-1.53\left(\mathrm{~m}, 6 \mathrm{H}, \mathrm{H}^{\mathrm{E}}\right), 1.79$ (quint, $2 \mathrm{H}, \mathrm{H}^{\mathrm{F}}$ ), $1.97\left(\mathrm{~m}\right.$ with fine coupling, $\left.3 \mathrm{H}, \mathrm{H}^{\mathrm{N}}\right), 3.91(\mathrm{t}$, $2 \mathrm{H}, \mathrm{H}^{\mathrm{G}}$ ), 5.59 ( $\mathrm{m}$ with fine coupling, $1 \mathrm{H}, \mathrm{H}^{\mathrm{O}}$ ), $6.20\left(\mathrm{~m}\right.$ with fine coupling, $\left.1 \mathrm{H}, \mathrm{H}^{\mathrm{P}}\right), 6.79(\mathrm{~d}$, $\left.2 \mathrm{H}, J=9.0 \mathrm{~Hz}, \mathrm{H}^{\mathrm{H}}\right), 7.13-7.42\left(\mathrm{~m}, 12 \mathrm{H}, \mathrm{H}^{\mathrm{I}}\right.$, $\left.\mathbf{H}^{\mathrm{J}}, \mathbf{H}^{\mathrm{K}}\right)$.

(4-Heptyloxyphenyl)diphenylmethyl Methacrylate $(\mathrm{R}=\mathrm{H}, m=4)(\mathrm{HpO})$.

$R f=0.26$ (hexane-ether, 4:1). Oil. Yield $83.3 \%$.

Chemical shift $\delta 0.88\left(\mathrm{t}, 3 \mathrm{H}, J=7.0 \mathrm{~Hz}, \mathrm{H}^{\mathrm{D}}\right)$, $1.15-1.54\left(\mathrm{~m}, 8 \mathrm{H}, \mathrm{H}^{\mathrm{E}}\right), 1.76$ (quint, $2 \mathrm{H}, \mathrm{H}^{\mathrm{F}}$ ), $1.98\left(\mathrm{~m}\right.$, with fine coupling, $\left.3 \mathrm{H}, \mathrm{H}^{\mathrm{N}}\right), 3.92(\mathrm{t}$, $2 \mathrm{H}, \mathrm{H}^{\mathrm{G}}$ ), 5.59 ( $\mathrm{m}$ with fine coupling, $1 \mathrm{H}, \mathrm{H}^{\mathrm{O}}$ ), $6.22\left(\mathrm{~m}\right.$ with fine coupling, $\left.1 \mathrm{H}, \mathrm{H}^{\mathrm{P}}\right), 6.80(\mathrm{~d}$, $\left.2 \mathrm{H}, J=9.0 \mathrm{~Hz}, \mathrm{H}^{\mathrm{H}}\right), 7.14-7.44\left(\mathrm{~m}, 12 \mathrm{H}, \mathrm{H}^{\mathbf{I}}\right.$, $\left.\mathbf{H}^{\mathrm{J}}, \mathbf{H}^{\mathbf{K}}\right)$.

(4-Octyloxyphenyl)diphenylmethyl Methacrylate $(\mathrm{R}=\mathrm{H}, m=5)(\mathrm{OcO})$.

$R f=0.23$ (hexane-ether, 5:1). Oil. Yield $90.0 \%$.

Chemical shift $\delta 0.88\left(\mathrm{t}, 3 \mathrm{H}, J=7.0 \mathrm{~Hz}, \mathrm{H}^{\mathrm{D}}\right)$, $1.20-1.54\left(\mathrm{~m}, 10 \mathrm{H}, \mathrm{H}^{\mathrm{E}}\right), 1.76$ (quint, $2 \mathrm{H}, \mathrm{H}^{\mathrm{F}}$ ), $1.98\left(\mathrm{~m}\right.$ with fine coupling, $\left.3 \mathrm{H}, \mathrm{H}^{\mathrm{N}}\right), 3.93(\mathrm{t}$, $2 \mathrm{H}, \mathrm{H}^{\mathrm{G}}$ ), $5.59\left(\mathrm{~m}\right.$ with fine coupling, $1 \mathrm{H}, \mathrm{H}^{\mathrm{O}}$ ), 6.22 (m with fine coupling, $\left.1 \mathrm{H}, \mathrm{H}^{\mathrm{P}}\right), 6.81(\mathrm{~d}$, $\left.2 \mathrm{H}, J=9.0 \mathrm{~Hz}, \mathrm{H}^{\mathrm{H}}\right), 7.14-7.45\left(\mathrm{~m}, 12 \mathrm{H}, \mathrm{H}^{\mathrm{I}}\right.$, $\left.\mathrm{H}^{\mathrm{J}}, \mathrm{H}^{\mathrm{K}}\right)$.

[4-\{3-(4-Methoxy-4'-oxybiphenyl)propoxy\}phenyldiphenylmethyl Methacrylate $(\mathrm{R}=4$ methoxy-4'-oxybiphenyl, $m=0$ ).

Twenty mole\% trimethylsilyl trifluoromethanesulfonate was used as catalyst. Recrystallized from ether.

Yield 40.5\%. mp n.d.

Chemical shifrs $\delta 1.98$ (m with fine coupling, $\left.3 \mathrm{H}, \mathrm{H}^{\mathrm{N}}\right), 2.25\left(\mathrm{t}, 2 \mathrm{H}, J=7.0 \mathrm{~Hz}, \mathrm{H}^{\mathrm{F}}\right), 3.84(\mathrm{~s}$, $\left.3 \mathrm{H}, \mathrm{H}^{\mathrm{A}}\right), 4.17\left(\mathrm{t}, 4 \mathrm{H}, \mathrm{H}^{\mathrm{D}}, \mathrm{H}^{\mathrm{G}}\right.$ ), 5.59 (m with fine coupling, $1 \mathrm{H}, \mathrm{H}^{\mathrm{O}}$ ), 6.23 (m with fine coupling, $1 \mathrm{H}, \mathrm{H}^{\mathrm{P}}$ ), 6.83, 6.94 (two $\mathrm{d}, 6 \mathrm{H}$, $\left.J=9.0 \mathrm{~Hz}, \mathrm{H}^{\mathrm{H}}, \mathrm{H}^{\mathrm{B}}\right), 7.19-7.28\left(\mathrm{~m}, 12 \mathrm{H}, \mathrm{H}^{\mathrm{I}}\right.$, $\left.\mathrm{H}^{\mathrm{J}}, \mathrm{H}^{\mathrm{K}}\right), 7.48\left(\mathrm{~d}, 4 \mathrm{H}, J=9.0 \mathrm{~Hz}, \mathrm{H}^{\mathrm{C}}\right)$.

[4-\{4-(4-Methoxy-4'-oxybiphenyl)butoxy\}phenyl]diphenylmethyl Methacrylate $(\mathrm{R}=4$ methoxy-4'-oxybiphenyl, $m=1$ ) (MBBuO).

Yield $42.7 \%$. mp $40.4^{\circ} \mathrm{C}$.

Chemical shifts $\delta 1.90-2.10\left(\mathrm{~m}, 7 \mathrm{H}, \mathrm{H}^{\mathrm{N}}, \mathrm{H}^{\mathrm{E}}\right.$, $\left.\mathrm{H}^{\mathrm{F}}\right), 3.85\left(\mathrm{~s}, 3 \mathrm{H}, \mathrm{H}^{\mathrm{A}}\right), 3.93-4.17\left(\mathrm{~m}, 4 \mathrm{H}, \mathrm{H}^{\mathrm{D}}\right.$, $\mathrm{H}^{\mathrm{G}}$ ), 5.61 (m with fine coupling, $1 \mathrm{H}, \mathrm{H}^{\mathrm{O}}$ ), 6.23 ( $\mathrm{m}$ with fine coupling, $1 \mathrm{H}, \mathrm{H}^{\mathrm{P}}$ ), 6.82, 6.95 (two $\left.\mathrm{d}, 6 \mathrm{H}, J=9.0 \mathrm{~Hz}, \mathrm{H}^{\mathrm{H}}, \mathrm{H}^{\mathrm{B}}\right), 7.13-7.32(\mathrm{~m}, 12 \mathrm{H}$, $\left.\mathrm{H}^{\mathrm{I}}, \mathrm{H}^{\mathrm{J}}, \mathrm{H}^{\mathrm{K}}\right), 7.47\left(\mathrm{~d}, 4 \mathrm{H}, J=9.0 \mathrm{~Hz}, \mathrm{H}^{\mathrm{C}}\right)$.

[4-\{5-(4-Methoxy-4'-oxybiphenyl)pentyloxy $\}$ phenyl $]$ diphenylmethyl Methacrylate $(\mathrm{R}=$ 4-methoxy-4'-oxybiphenyl, $m=2$ ) (MBPeO).

Yield $69.9 \%$. mp $39.2^{\circ} \mathrm{C}$.

Chemical shifts $\delta 1.59-1.96\left(\mathrm{~m}, 6 \mathrm{H}, \mathrm{H}^{\mathrm{E}}\right.$, $\left.\mathrm{H}^{\mathrm{F}}\right), 1.99\left(\mathrm{~m}\right.$, with fine coupling, $\left.3 \mathrm{H}, \mathrm{H}^{\mathrm{N}}\right), 3.84$ (s, 3H, $\mathrm{H}^{\mathrm{A}}$ ), 3.98, 4.02 (two t, $4 \mathrm{H}, \mathrm{H}^{\mathrm{D}}, \mathrm{H}^{\mathrm{G}}$ ), $5.60\left(\mathrm{~m}\right.$, with fine coupling, $\left.1 \mathrm{H}, \mathrm{H}^{\mathrm{O}}\right), 6.23(\mathrm{~m}$ with fine coupling, $1 \mathrm{H}, \mathrm{H}^{\mathrm{P}}$ ), 6.81, 6.95 (two d, $\left.6 \mathrm{H}, J=9.0 \mathrm{~Hz}, \mathrm{H}^{\mathrm{H}}, \mathrm{H}^{\mathrm{B}}\right), 7.13-7.33(\mathrm{~m}, 12 \mathrm{H}$, $\left.\mathrm{H}^{\mathrm{I}}, \mathrm{H}^{\mathrm{J}}, \mathrm{H}^{\mathrm{K}}\right), 7.47\left(\mathrm{~d}, 4 \mathrm{H}, J=9.0 \mathrm{~Hz}, \mathrm{H}^{\mathrm{C}}\right)$.

\section{Polymerization}

Monomers other than triphenylmethyl methacrylate were used after drying with calcium hydride as toluene solution followed by filtration. Triphenylmethyl methacrylate was purified by recrystallization. Radical polymerizations were carried out in toluene $(1 \mathrm{~g}$ monomer $/ 6 \mathrm{ml}$ solvent) at $60^{\circ} \mathrm{C}$ with $2 \mathrm{~mol} \%$ AIBN as an initiator for $72 \mathrm{~h}$. Anionic polymerizations were initiated by an alkyllithium-amine complex system using butyllithium as an alkyllithium component. Polymers were recovered by pouring the polymerization system into methanol.

\section{Conversion of Polymers into Poly(methyl methacrylate) $s^{1,7}$}

Polymers were hydrolyzed by methanolic hydrochloric acid by heating the mixture for $24 \mathrm{~h}$. The hydrolyzed poly(methacrylic acid)s 
were quantitatively recovered by pouring into ether. Thus obtained poly(methacrylic acid)s were esterified with diazomethane to give poly(methyl methacrylate)s. Polymers were quantitatively recovered by pouring into hexane.

\section{Molecular Weight of Polymers and Triad Tacticity}

Molecular weight data were estimated by gel permeation chromatography using polystyrene standard and tacticity was determined by NMR after converting into poly(methyl methacrylate)s.

\section{RESULTS AND DISCUSSION}

\section{Synthesis of Monomers}

The minimum lengths of the alkyl groups as tail group to stabilize the helical structure of poly $(\gamma$-alkyl-L-glutamate)s, poly( $\gamma$-benzyl-Lglutamate-co- $\gamma$-alkyl-L-glutamate)s or $\operatorname{poly}(\gamma$ methyl-D-glutamate-co- $\gamma$-alkyl-D-glutamate)s are 10,12 or 6 (difference in length of alkyl groups is lager than 5) in order to exhibit thermotropic liquid crystalline state. ${ }^{6,8,9} \mathrm{Judg}$ ing from above mentioned fact, triphenylmethyl methacrylates with tail groups longer than 5 were attempted to synthesize, because isotactic polymers of these monomers might take thermotropic liquid crystalline state under proper conditions.

Synthesis of triphenylmethyl methacrylate monomer was reported by several authors. Adrova $^{10}$ and Berlin ${ }^{11}$ reported the synthesis by the reaction of triphenylmethyl chloride with alkaline metal or silver salt of methacrylic acid. Monomers were recrystallized from hexane after removing the formed salt. However, monomer thus obtained contained some amounts of salt, and specially monomer from silver salt was easily colored by light. Moreover, these reactions are often not satisfactory in the synthesis of analogous methacrylate compounds. By the search for the reaction conditions, a modified method re- ported by Noyori, ${ }^{12}$ in which trimethylsilyl ether of triphenylmethyl carbinol was reacted with trimethylsilyl methacrylate in the presence of trimethylsilyl trifluoromethanesulfonate, was found to give satisfactory results. Since the reagents were unstable against hydrolysis, caution must be taken to avoid the compounds in contact with water as much as possible. The products mono-p-alkoxy-substituted triphenylmethyl methacrylates are also easily hydrolyzed during the separation procedure or purification step. In purifying these compounds by column chromatography, use of well dried $\left(120^{\circ} \mathrm{C}\right.$ for $\left.5 \mathrm{~h}\right)$ silica gel and solvent, and chromatography at $0^{\circ} \mathrm{C}$ is recommended.

\section{Polymerization Conditions and Reactivity of Monomer}

Results of the radical polymerization are shown in Table I. In Table I, the molecular weight of polymers are shown as those for poly(methyl methacrylate)s [see the section of Conversion of Polymers into Poly(methyl methacrylate)]. The results of anionic polymerization are shown in Table II.

Reasonable yield and degree of polymerization could be obtained by radical polymerization. This fact guarantees the intrinsic polymerizability of monomers in thermodynamic sense.

The polymers [non substituted or short alkoxy (methoxy, ethoxy, propoxy, and butoxy) substituted poly(triphenylmethyl methacrylate)] are insoluble in ordinary solvents. The solubility of polymers increased with the length of alkoxy substituents. Hexyloxy substituted polymers are even soluble in toluene, chloroform and tetrahydrofuran.

Considerable amounts of initiator were required to bring the reaction system to polymerize by anionic initiators. ${ }^{13}$ Although triphenylmethyl methacrylate and methyl substituted triphenylmethyl methacrylate gave reasonable yield even by anionic initiator, methoxy or butoxy substituted monomers gave only poor yield of low molecular weight 
Table I. Radical polymerization of mono-p-alkoxy-substituted triphenylmethyl methacrylates and molecular weight data estimated as poly(methyl methacrylate)s

\begin{tabular}{|c|c|c|c|c|c|}
\hline \multirow{2}{*}{ Monomer } & \multirow{2}{*}{$\begin{array}{c}\text { State of } \\
\text { polym'n system }\end{array}$} & \multirow{2}{*}{$\begin{array}{c}\text { Yield } \\
\%\end{array}$} & \multicolumn{3}{|c|}{ PMMA } \\
\hline & & & $M_{w} \times 10^{3}$ & $M_{n} \times 10^{3}$ & $M_{w} / m_{n}$ \\
\hline $\operatorname{TrMA}_{\text {anionic }}$ & Heterogeneous & 94.7 & 12.2 & 8.4 & 1.4 \\
\hline $\operatorname{TrMA}_{\text {radical }}$ & Heterogeneous & 81.3 & 37.5 & 18.4 & 2.0 \\
\hline $\mathrm{MeO}$ & Heterogeneous & 46.5 & 37.8 & 20.6 & 1.8 \\
\hline EtO & Heterogeneous & 76.3 & 12.6 & 6.2 & 2.0 \\
\hline $\mathrm{PrO}$ & Heterogeneous & 67.9 & 17.3 & 12.4 & 1.4 \\
\hline $\mathrm{BuO}$ & Heterogeneous & 33.8 & 19.2 & 10.8 & 1.8 \\
\hline $\mathrm{PeO}$ & Heterogeneous & 34.9 & 15.0 & 10.1 & 1.5 \\
\hline $\mathrm{HxO}$ & Homogeneous & 60.3 & 16.8 & 11.6 & 1.5 \\
\hline $\mathrm{HpO}$ & Homogeneous & 71.2 & 25.5 & 17.7 & 1.4 \\
\hline $\mathrm{OcO}$ & Homogeneous & 46.2 & 19.1 & 8.4 & 2.3 \\
\hline $\mathrm{MBBuO}$ & Heterogeneous & 55.9 & 24.5 & 14.3 & 1.7 \\
\hline MBPeO & Heterogeneous & 6.0 & - & - & - \\
\hline
\end{tabular}

Initiator: $\mathrm{AIBN},[\mathrm{M}] /[\mathrm{I}]=50,60^{\circ} \mathrm{C}, 120 \mathrm{~h}$ in toluene.

Molecular weights are estimated by gel permeation chromatography correlating to standard polystyrene after converting into poly(methyl methacrylate).

Table II. Anionic polymerization of mono-p-alkoxy-substituted triphenylmethyl methacrylates

\begin{tabular}{|c|c|c|c|c|c|c|c|}
\hline \multirow{3}{*}{ Monomer } & \multirow{3}{*}{ Initiator } & \multirow{3}{*}[\mathrm{M}]{$/[\mathrm{I}]$} & \multirow{3}{*}{$\frac{\text { Time }}{\mathrm{h}}$} & \multicolumn{4}{|c|}{ Polymer } \\
\hline & & & & Yield & & & \\
\hline & & & & $\%$ & & & \\
\hline TrMA & $(-) \mathrm{DDB}-\mathrm{BuLi}$ & 8.9 & 3 & 94.7 & 33 & 18.5 & 1.8 \\
\hline $\mathrm{MeO}$ & $t$-BuLi & 10.0 & 24 & 12.6 & 2.5 & 1.2 & 2.1 \\
\hline $\mathrm{BuO}$ & $(-) \mathrm{DDB}-\mathrm{BuLi}$ & 2.0 & 24 & 15.4 & 3.3 & 2.9 & 1.2 \\
\hline $\mathrm{Me}$ & (-)DDB-FlLi & 10.0 & 48 & 43.8 & 10.8 & 2.1 & 5.2 \\
\hline
\end{tabular}

Polymerizations were carried out in toleuene at $-78^{\circ} \mathrm{C}$.

Molecular weights of the formed polymers are estimated by gel permeation chromatography without converting into poly(methyl methacrylate) correlating to standard polystyrene. Because of the low yield or wide distribution of molecular weight, optical rotation of the polymer was not measured.

polymers. With lower concentration of initiator than those shown in Table II, polymerization hardly proceeded. Addition of triethylaluminum ${ }^{14}$ did not improve the yield, nor degree of polymerization. The low yield and low degree of polymerization by anionic initiators are attributed to either the presence of impurity in monomer or instability of monomers against nucleophilic attack by initiator or propagating anion to lead to side reactions.

\section{Stability of Monomers and Polymers against} Hydrolysis

Although alkoxy substituted monomers were labile to hydrolysis, column chromatography using well dried silica gel and solvent gave pure monomer without significant amounts of decomposition. Such instability of the mono p-alkoxy substituted monomers against hydrolysis seemed to be brought about by the presence of the ether linkage at $p$ - 
position. Okamoto et al. reported ${ }^{15}$ the stability of substituted triphenylmethyl methacrylates, and showed the relationship between the rate of the methanolysis and Hammet's $\sigma^{+}$. Electron with drawing substitutents seem to stabilize the monomer against hydrolysis.

Methyl substituted monomer is considerably stable against hydrolysis. Polymers are usually much stabler than monomers against hydrolysis. No indication of hydrolysis was observed in the reprecipitation procedures in methanol. Hydrophobic environment of polymer back bone may protect the labile ether linkage from cleavage.

\section{Streoregularity of Polymers}

The tacticities of polymers are shown in Table III. The diads determined from NMR spectra are fairly in good accordance with the calculated probability values of isotactic propagation $\left(\boldsymbol{P}_{\mathrm{m}}\right)$ based on general relations between triad and diad. ${ }^{16}$ In contrast to the fact that radical polymerization, in general, gives syndiotactic polymer from methyl methacrylate, ${ }^{17}$ polymers with considerably high isotactic triad are obtained even by radical polymerization.

Niezette ${ }^{1}$ and $\mathrm{Yuki}^{13}$ reported on the isotacticity of polymethacrylates with bulky ester residue groups. Contrary to the fact that $t$-butyl methacrylate or ordinary aromatic methacrylates gave polymers whose tacticity was comparable with that of poly(methyl methacrylate) formed by radical initiators, triphenylmethyl methacrylate gave polymers with considerably high isotacticity even by radical method.

Probabilities characterizing the stereochemistry of the propagation as first-order Markov process are also shown in Table III. Discussion about the stereoregularity of polymers is made based on the triad tacticity because separation of triad peaks is much better than that of the diad peaks. The sum of the probability values $\left(P_{\mathrm{m} / \mathrm{r}}+P_{\mathrm{r} / \mathrm{m}}\right)$ are much smaller than unity. This fact indicates that the statistic ruling this polymerization does not obey Bernoulli trial (in case Bernoullian, this value should be unity).

Niezette suggested special interaction between phenyl moieties in the $3_{1}$ helical structure of the propagating chain stabilized by polar and steric effects as cited in Figure 1. This suggestion may imply the importance of the interaction between phenyl groups in monomer and penultimate unit in controlling the

Table III. Tacticity of the polymers prepared by radical polymerization estimated as poly(methyl methacrylate)

\begin{tabular}{|c|c|c|c|c|c|c|c|c|c|}
\hline \multirow{3}{*}{$\begin{array}{l}\text { Starting } \\
\text { polymer }\end{array}$} & \multicolumn{5}{|c|}{ Tacticity (measured) } & \multicolumn{4}{|c|}{ Probability (calculated) } \\
\hline & \multicolumn{3}{|c|}{ Triad $/ \%$} & \multicolumn{2}{|c|}{$\operatorname{Diad} / \%$} & \multirow[b]{2}{*}{$P_{\mathrm{m}}$} & \multirow[b]{2}{*}{$P_{\mathrm{m} / \mathrm{r}}$} & \multirow[b]{2}{*}{$P_{\mathrm{r} / \mathrm{m}}$} & \multirow[b]{2}{*}{$P_{\mathrm{m} / \mathrm{r}}+P_{\mathrm{r} / \mathrm{m}}$} \\
\hline & I & $\mathbf{H}$ & $\mathrm{S}$ & $i$ & $s$ & & & & \\
\hline PTrMA $_{\text {anionic }}$ & 92 & 4 & 4 & 95 & 6 & 0.94 & 0.02 & 0.33 & 0.35 \\
\hline PTrMA & 72 & 16 & 12 & 79 & 20 & 0.80 & 0.10 & 0.40 & 0.50 \\
\hline $\mathrm{PMeO}$ & 52 & 31 & 17 & 63 & 32 & 0.68 & 0.23 & 0.48 & 0.71 \\
\hline PEtO & 61 & 24 & 14 & 70 & 26 & 0.73 & 0.16 & 0.46 & 0.63 \\
\hline PPrO & 69 & 23 & 7 & 76 & 19 & 0.81 & 0.14 & 0.62 & 0.76 \\
\hline PBuO & 84 & 10 & 6 & 88 & 11 & 0.89 & 0.06 & 0.45 & 0.51 \\
\hline $\mathrm{PPeO}$ & 83 & 12 & 5 & 83 & 11 & 0.89 & 0.07 & 0.55 & 0.61 \\
\hline $\mathrm{PHxO}$ & 65 & 23 & 12 & 70 & 24 & 0.77 & 0.15 & 0.49 & 0.64 \\
\hline PHpO & 65 & 22 & 13 & 72 & 24 & 0.76 & 0.14 & 0.46 & 0.60 \\
\hline $\mathrm{POcO}$ & 75 & 13 & 12 & 80 & 18 & 0.82 & 0.08 & 0.35 & 0.43 \\
\hline PMBBuO & 71 & 15 & 14 & 78 & 21 & 0.79 & 0.10 & 0.35 & 0.44 \\
\hline
\end{tabular}




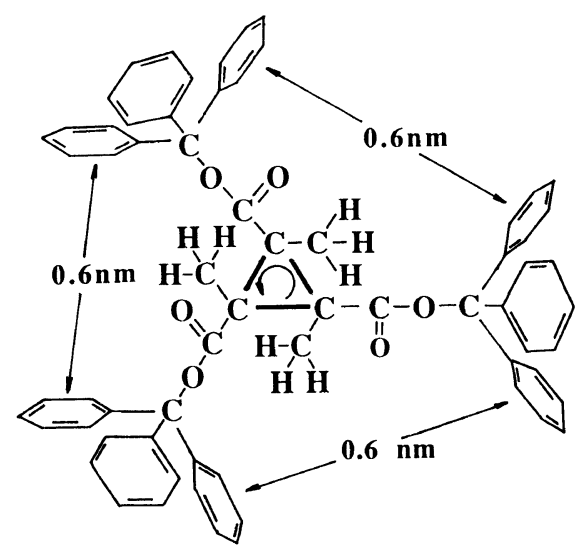

Figure 1. Interaction of phenyl groups along the $3_{1}$ helical polymer back bone suggested by Niezette. ${ }^{1}$

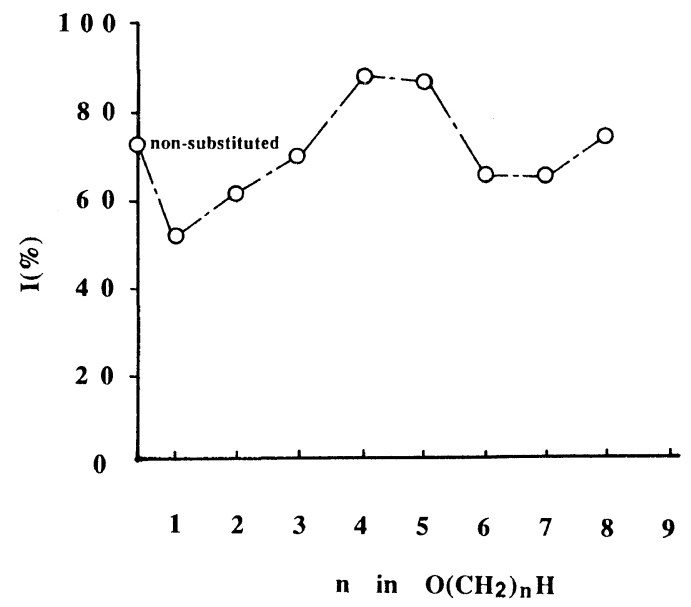

Figure 2. Plot of the change in triad isotacticity with the change of alkoxy group at one of the p-position of poly(triphenylmethyl methacrylate).

direction of coordination of monomer to growing end.

The effects of the length of the $p$-substituents on the triad isotacticity of polymers obtained by radical initiator are shown in Figure 2 . Evidently, the length of the alkoxy groups has significant effects on the isotacticity.

In alkoxy substituted triphenylmethyl methacrylate now studied, methoxy groups lowered the tacticity. Apparently steric effects of the methoxy groups prohibit such stabilization by the interaction through phenyl groups.
The isotacticity again increased with the length of the alkoxy substituents. Maximum in tacticity was observed for butoxy or pentyloxy substituents. Alkoxy groups of middle length may stabilize such interaction by polar effects. The tacticity of polymers with longer alkoxy substituents seems to decrease again and gradually reach to a plateau value. Although the reason is not clear, steric and polar effects may work irreconcilably in stabilizing such interaction between the phenyl groups at penultimate unit and monomer, and finally balances with longer alkoxy substituents. Detailed research is now in progress.

\section{Polymers with Mesogenic Groups}

Monomers with mesogenic groups can be also polymerized, although the yield seems to be lower than that of monomers without mesogenic groups. Poly(MBBuO) showed considerably high triad isotacticity for a radical polymerized polymer. Poly(MBBuO) and poly $(\mathrm{MBPeO})$ showed endothermic peaks at 54 and $46^{\circ} \mathrm{C}$, respectively in DSC analysis. These polymers gave spherulite structure in microscopic observation. Further study will be published elsewhere.

Acknowledgment. The authors express their sincere thanks to the Financial supports from a Grant-in-Aid for Developmental Scientific Research (No. 63850184) and from a Grnat-in-Aid for Scientific Research on Priority Areas, New Functionality MaterialsDesign, Preparation and Control (No. 01604551), the Ministry of Education, Science, and Culture of Japan.

\section{REFERENCES}

1. J. Niezette and V. Desreux, Makromol. Chem., 149, 177 (1971).

2. Y. Okamoto, K. Suzuki, K. Ohta, K. Hatada, and H. Yuki, J. Am. Chem. Soc., 101, 4763 (1979).

3. H. Yuki, Y. Okamoto, and I. Okamoto, J. Am. Chem. Soc., 102, 6356 (1980).

4. Y. Okamoto and T. Nakano, J. Synth. Org. Chem. 
(Yuki Gosei Kagaku Kyokaishi) (in Japanese), 47, 1029 (1989).

5. I. Uematsu and Y. Uematsu, Adv. Polym. Sci., 59, 37 (1984).

6. J. Watanabe, H. Ono, I. Uematsu, and A. Abe, Macromolecules, 18, 2141 (1985).

7. A. Katchalsky and H. Eisenberg, J. Polym. Sci., 6, 145 (1951).

8. J. Watanabe, Y. Fukuda, R. Gehani, and I. Uematsu, Macromolecules, 17, 1004 (1984).

9. J. Watanabe, M. Goto, and T. Nagase, Macromolecules, 20, 298 (1987).

10. N. A. Adrova and K. K. Prokhorova, Vysokomol. Soed., 3, 1509, (1961).
11. K. D. Berlin, K. H. Gower, J. W. White, D. E. Gibbs, and G. P. Sturm, J. Org. Chem., 27, 3595 (1962).

12. S. Murata and R. Noyori, Tetrahedron Lett., 22, 2107 (1981).

13. H. Yuki, K. Hatada, Y. Kikuchi, and T. Niinomi, Polym. Lett., 6, 753 (1968).

14. H. Yuki, K. Hatada, Y. Kikuchi, and T. Niinomi, Polym. Lett., 6, 753 (1968).

15. Y. Okamoto, E. Yashima, M. Ishikura, and K. Hatada, Polym. J., 19, 1183 (1987).

16. F. A. Bovey, "Chain Structure and Conformation of Macromolecules," Academic Press, New York, 1982.

17. F. A. Bovey, J. Polym. Sci., 46, 59 (1960). 